TRANSACTIONS OF THE

AMERICAN MATHEMATICAL SOCIETY

Volume 362, Number 5, May 2010, Pages 2641-2666

S 0002-9947(09)05023-5

Article electronically published on December 3, 2009

\title{
AUTOMORPHISM GROUPS ON NORMAL SINGULAR CUBIC SURFACES WITH NO PARAMETERS
}

\author{
YOSHIYUKI SAKAMAKI
}

\begin{abstract}
The classification of normal singular cubic surfaces in $\mathbf{P}^{3}$ over a complex number field $\mathbf{C}$ was given by J. W. Bruce and C. T. C. Wall. In this paper, first we prove their results by a different way, second we provide normal forms of normal singular cubic surfaces according to the type of singularities, and finally we determine automorphism groups on normal singular cubic surfaces with no parameters.
\end{abstract}

\section{INTRODUCTION}

The study of a classification of complex projective cubic surfaces in $\mathbf{P}^{3}$ by their singularities was begun by L. Schläfli [16] and A. Cayley [3] in the nineteenth century. In the 1970s, J. W. Bruce and C. T. C. Wall [2] classified normal singular cubic surfaces by modern singularity terminology using the Dynkin diagram (Theorem 1). On the other hand, automorphism groups on nonsingular cubic surfaces were determined by B. Segre [17, T. Hosoh [9] and so forth. Recently, automorphisms of smooth cubic surfaces in $\mathbf{P}^{3}$ were used by I. V. Dolgachev and V. A. Iskovskikh [6] in their study on classification of conjugacy classes of finite subgroups of the plane Cremona group, which is the group of birational automorphisms of the projective plane.

In this paper we consider automorphism groups on normal singular cubic surfaces. First we prove the results of Bruce and Wall (Theorem 1) by a method which is different from the one of [2], second we give normal forms of normal singular cubic surfaces according to type of singularities (Theorem 2), and finally we determine automorphism groups on normal singular cubic surfaces which have no parameters in their normal forms (Theorem 31).

First we recall the classification of normal singular cubic surfaces.

Theorem 1 (J. W. Bruce, C. T. C. Wall [2]). Any normal singular cubic surface in $\mathbf{P}^{3}$ has either rational double points or a simple elliptic singularity $\tilde{E}_{6}$ in Table 1. Moreover, the number of parameters and the number of lines on the surface, according to type of singularities, are listed in Table 1.

Our main results are Theorems 2 and 3. Normal forms of singular cubic surfaces which have $D_{4}, D_{5}, E_{6}$ singularity were given in [2]. Theorem 2 provides normal forms of all normal singular cubic surfaces.

Received by the editors November 13, 2007 and, in revised form, July 29, 2008.

2010 Mathematics Subject Classification. Primary 14J50; Secondary 14J17.

Key words and phrases. Algebraic geometry, automorphism groups, cubic surfaces, singularities, blowing-up, infinitely near points.

(C)2009 American Mathematical Society Reverts to public domain 28 years from publication 
TABLE 1

\begin{tabular}{|c|c|c|c|c|c|c|c|c|c|c|c|c|c|}
\hline \multicolumn{3}{|c|}{ Singularities } & $A_{1}$ & $2 A_{1}$ & $A_{1}$ & & $3 A_{1}$ & $A_{1} A_{3}$ & \multicolumn{2}{|c|}{$2 A_{1} A_{2}$} & $4 A_{1}$ & \multicolumn{2}{|c|}{$A_{1} A_{4}$} \\
\hline \multicolumn{3}{|c|}{ No.of parameters } & 3 & 2 & & & 1 & 0 & \multicolumn{2}{|c|}{0} & 0 & \multicolumn{2}{|c|}{0} \\
\hline \multicolumn{3}{|c|}{ No.of lines } & 21 & 16 & 1 & & 12 & 7 & \multicolumn{2}{|c|}{8} & 9 & \multicolumn{2}{|c|}{4} \\
\hline $2 A_{1} A_{3}$ & $\overline{A_{1} 2 A_{2}}$ & $A_{1} A$ & & $\overline{A_{2}}$ & $2 A_{2}$ & $3 A$ & $\overline{A_{3}}$ & $\overline{A_{4}}$ & $A_{5}$ & $D_{4}$ & $\overline{D_{5}}$ & $\overline{E E_{6}}$ & $\tilde{E}_{6}$ \\
\hline 0 & 0 & 0 & & 2 & 1 & 0 & 1 & 0 & 0 & 0 & 0 & 0 & 1 \\
\hline 5 & 5 & 2 & & 15 & 7 & 3 & 10 & 6 & 3 & 6 & 3 & 1 & $\infty$ \\
\hline
\end{tabular}

TABLE 2

\begin{tabular}{ccc}
\hline Singularities & $f_{2}\left(x_{0}, x_{1}, x_{2}\right)$ & $f_{3}\left(x_{0}, x_{1}, x_{2}\right)$ \\
\hline$A_{1}$ & $x_{0} x_{2}-x_{1}^{2}$ & $\left(x_{0}-a x_{1}\right)\left(-x_{0}+(b+1) x_{1}-b x_{2}\right)\left(x_{1}-c x_{2}\right)$ \\
$2 A_{1}$ & $x_{0} x_{2}-x_{1}^{2}$ & $\left(x_{0}-2 x_{1}+x_{2}\right)\left(x_{0}-a x_{1}\right)\left(x_{1}-b x_{2}\right)$ \\
$A_{1} A_{2}$ & $x_{0} x_{2}-x_{1}^{2}$ & $\left(x_{0}-x_{1}\right)\left(-x_{1}+x_{2}\right)\left(x_{0}-(a+1) x_{1}+a x_{2}\right)$ \\
$3 A_{1}$ & $x_{0} x_{2}-x_{1}^{2}$ & $x_{0} x_{2}\left(x_{0}-(a+1) x_{1}+a x_{2}\right)$ \\
$A_{1} A_{3}$ & $x_{0} x_{2}-x_{1}^{2}$ & $\left(x_{0}-x_{1}\right)\left(-x_{1}+x_{2}\right)\left(x_{0}-2 x_{1}+x_{2}\right)$ \\
$2 A_{1} A_{2}$ & $x_{0} x_{2}-x_{1}^{2}$ & $x_{1}^{2}\left(x_{0}-x_{1}\right)$ \\
$4 A_{1}$ & $x_{0} x_{2}-x_{1}^{2}$ & $\left(x_{0}-x_{1}\right)\left(x_{1}-x_{2}\right) x_{1}$ \\
$A_{1} A_{4}$ & $x_{0} x_{2}-x_{1}^{2}$ & $x_{0}^{2} x_{1}$ \\
$2 A_{1} A_{3}$ & $x_{0} x_{2}-x_{1}^{2}$ & $x_{0} x_{1}^{2}$ \\
$A_{1} 2 A_{2}$ & $x_{0} x_{2}-x_{1}^{2}$ & $x_{1}^{3}$ \\
$A_{1} A_{5}$ & $x_{0} x_{2}-x_{1}^{2}$ & $x_{0}^{3}$ \\
$A_{2}$ & $x_{0} x_{1}$ & $x_{2}\left(x_{0}+x_{1}+x_{2}\right)\left(d x_{0}+e x_{1}-d e x_{2}\right)$ \\
$2 A_{2}$ & $x_{0} x_{1}$ & $x_{2}\left(x_{1}+x_{2}\right)\left(-x_{1}+d x_{2}\right)$ \\
$3 A_{2}$ & $x_{0} x_{1}$ & $x_{2}^{3}$ \\
$A_{3}$ & $x_{0} x_{1}$ & $x_{2}\left(x_{0}+x_{1}+x_{2}\right)\left(x_{0}-u x_{1}\right)$ \\
$A_{4}$ & $x_{0} x_{1}$ & $x_{0}^{2} x_{2}+x_{1}^{3}-x_{1} x_{2}^{2}$ \\
$A_{5}$ & $x_{0} x_{1}$ & $x_{0}^{3}+x_{1}^{3}-x_{1} x_{2}^{2}$ \\
$D_{4}(1)$ & $x_{0}^{2}$ & $x_{1}^{3}+x_{2}^{3}$ \\
$D_{4}(2)$ & $x_{0}^{2}$ & $x_{1}^{3}+x_{2}^{3}+x_{0} x_{1} x_{2}$ \\
$D_{5}$ & $x_{0}^{2}$ & $x_{0} x_{2}^{2}+x_{1}^{2} x_{2}$ \\
$E_{6}$ & $x_{0}^{2}$ & $x_{0} x_{2}^{2}+x_{1}^{3}$ \\
$\tilde{E}_{6}$ & 0 & $x_{1}^{2} x_{2}-x_{0}\left(x_{0}-x_{2}\right)\left(x_{0}-a x_{2}\right)$ \\
\hline
\end{tabular}

Theorem 2. Let $X$ be a normal singular cubic surface in $\mathbf{P}^{3}$. Then $X$ is isomorphic to the projective surface in $\mathbf{P}^{3}$ defined by $F=x_{3} f_{2}\left(x_{0}, x_{1}, x_{2}\right)-f_{3}\left(x_{0}, x_{1}, x_{2}\right)=$ 0 , where $f_{2}, f_{3}$ are in Table 2, according to type of singularities on $X$. In Table 2 , $a, b, c$ are three distinct elements of $\mathbf{C} \backslash\{0,1\}, d$, e are elements of $\mathbf{C} \backslash\{0,-1\}$, and $u$ is an element of $\mathbf{C}^{\times}:=\mathbf{C} \backslash\{0\}$. (The polynomial $F$ is called the normal form of $X$.)

By studying automorphism groups on normal singular cubic surfaces which have no parameters in their normal forms in Table 2, we get the following.

Theorem 3. Let $X$ be a normal singular cubic surface in $\mathbf{P}^{3}$ which has no parameters in the normal form in Table 2. Then the automorphism group on $X$ (Aut $X$, for short) is given in Table 3 according to the type of singularities on $X$, where $\Sigma_{n}$ is a symmetric group of degree $n$.

The structure of this paper is as follows. In section 1, we will see that any rational normal singular cubic surface $X$ in $\mathbf{P}^{3}$ corresponds to two effective divisors $C_{2}, C_{3}$ on $\mathbf{P}^{2}$, where $\operatorname{deg} C_{i}=i$, and we prepare the notation of intersection symbol for $C_{2}$ and $C_{3}$ (see Definition 1.2). Letting $\varepsilon: \tilde{X} \rightarrow \mathbf{P}^{2}$ be the composite of blowings-up at six (possibly infinitely near) points which are intersection points of $C_{2}$ and $C_{3}$, 
TABLE 3

\begin{tabular}{cc}
\hline Singularities & Aut $X$ \\
\hline$A_{1} A_{3}$ & $\mathbf{Z} / 2 \mathbf{Z}$ \\
$2 A_{1} A_{2}$ & $\mathbf{Z} / 2 \mathbf{Z}$ \\
$4 A_{1}$ & $\Sigma_{4}$ \\
$A_{1} A_{4}$ & $\mathbf{C}^{\times}$ \\
$2 A_{1} A_{3}$ & $\mathbf{C}^{\times} \rtimes \mathbf{Z} / 2 \mathbf{Z}$ \\
$A_{1} 2 A_{2}$ & $\mathbf{C} \rtimes \mathbf{Z} / 2 \mathbf{Z}$ \\
$A_{1} A_{5}$ & $\mathbf{C} \rtimes \mathbf{C}^{\times}$ \\
$3 A_{2}$ & $\left(\mathbf{C}^{\times}\right)^{2} \rtimes \Sigma_{3}$ \\
$A_{4}$ & $\mathbf{Z} / 4 \mathbf{Z}$ \\
$A_{5}$ & $(\mathbf{C} \rtimes \mathbf{Z} / 3 \mathbf{Z}) \rtimes \mathbf{Z} / 2 \mathbf{Z}$ \\
$D_{4}(1)$ & $\mathbf{C} \rtimes \Sigma_{3}$ \\
$D_{4}(2)$ & $\Sigma_{3}$ \\
$D_{5}$ & $\mathbf{C}^{\times}$ \\
$E_{6}$ & $\mathbf{C} \rtimes \mathbf{C}^{\times}$ \\
\hline
\end{tabular}

we obtain a birational morphism $\mu: \tilde{X} \rightarrow X$ which gives the minimal resolution of singularities, and we prove that $X$ has only rational double points. In section 2. we will determine all $(-1)$-curves and $(-2)$-curves on $\tilde{X}$ with respect to each intersection symbol. We denote by $\Gamma_{\tilde{X}}$ the set of all $(-1),(-2)$-curves on $\tilde{X}$. It is a finite set in any case. We classify rational normal singular cubic surfaces by the Dynkin diagram which is given by the configuration of $(-2)$-curves, and we determine the number of lines on their surfaces (Theorem 11). In section 3, for rational normal singular cubic surfaces, we will have the normal forms and the number of parameters with respect to each type of singularity (Theorems 10, 2). In section 4 , we first prove that Aut $X$ is isomorphic to Aut $\tilde{X}$. Finally we have automorphism groups on cubic surfaces which have no parameters in their normal forms (Theorem 3). There exists a group homomorphism $\Psi:$ Aut $\tilde{X} \rightarrow \operatorname{Aut} \Gamma_{\tilde{X}}$, where Aut $\Gamma_{\tilde{X}}$ is the automorphism group of configuration of $\Gamma_{\tilde{X}}$ (see Definition 4.2). For a normal singular cubic surface with no parameters, we can show that $\Psi$ is surjective with respect to each type of singularity, and we have a short exact sequence,

$$
0 \longrightarrow \operatorname{Ker} \Psi \longrightarrow \text { Aut } \tilde{X} \longrightarrow \text { Aut } \Gamma_{\tilde{X}} \longrightarrow 0 \text {. }
$$

Aut $\Gamma_{\tilde{X}}$ is determined by intersection relations of $(-1),(-2)$-curves on $\tilde{X}$, and $\operatorname{Ker} \Psi$ is isomorphic to a subgroup of Aut $\mathbf{P}^{2}$. Therefore, we can determine the automorphism groups on normal singular cubic surfaces with no parameters.

Terminology and notation. For the most part, the terminology and notation of this paper agree with generally accepted usage. Throughout this paper, a surface will mean a projective surface over a complex number field $\mathbf{C}$.

For a smooth surface $S$, we denote the canonical sheaf by $\omega_{S}$, the canonical divisor by $K_{S}$, the Picard group by Pic $S$, and the free abelian group generated by prime divisors by $\operatorname{Div} S$. For two divisors $C, D$ on $S$, we denote the intersection number of $C$ and $D$ by $C . D$, the intersection multiplicity of $C$ and $D$ at a point $P \in S$ by $(C . D)_{P}$, and the multiplicity of $C$ at a point $P \in S$ by $\mu_{P}(C)$. We write $C \sim D$ if $C$ and $D$ are linearly equivalent. 


\section{The minimal Resolution of Singularities OF A NORMAL SINGULAR CUBIC SURFACE}

1.1. Intersection symbol. Let $X \subset \mathbf{P}^{3}$ be a normal singular cubic surface and let $P$ be a singular point on $X .\left(x_{0}: x_{1}: x_{2}: x_{3}\right)$ denotes a homogeneous coordinate in $\mathbf{P}^{3}$. By a projective transformation sending $P$ to $(0: 0: 0: 1), X$ is isomorphic to a projective surface in $\mathbf{P}^{3}$ defined by

$$
x_{3} f_{2}\left(x_{0}, x_{1}, x_{2}\right)-f_{3}\left(x_{0}, x_{1}, x_{2}\right)=0,
$$

where $f_{i}$ is a homogeneous polynomial of degree $i(i=2,3)$. The rank of the quadratic form $f_{2}$ (rank $f_{2}$, for short) is three or less, and it does not depend on a choice of the transformation above.

Remark 1.1. If rank $f_{2}$ is zero, then $X$ is projectively equivalent to a cone by a smooth cubic curve in $\mathbf{P}^{2}$, so $X$ contains infinitely many lines. Moreover, $X$ has the only simple elliptic singularity $\tilde{E}_{6}$.

Hereafter, let $X$ be a normal singular cubic surface defined by the equation $(\natural)$, and we suppose that $\operatorname{rank} f_{2}$ is not zero. We regard $C_{2}:=\left\{f_{2}=0\right\}$ and $C_{3}:=\left\{f_{3}=0\right\}$ as effective divisors on the projective plane $\mathbf{P}^{2}$. Modifying slightly the usage of [2], we define the intersection symbol below.

Definition 1.2. If rank $f_{2}=3$, then $C_{2}$ is a smooth conic. For $i=1, \ldots, 6$, let $m_{i}$ be the number of points $Q$ with $\left(C_{2} \cdot C_{3}\right)_{Q}=i$. Then the notation $6^{m_{6}} 5^{m_{5}} \cdots 1^{m_{1}}$ is called the intersection symbol of $C_{2}$ and $C_{3}$. However, if $m_{i}=0$ or $m_{i}=1$, then we omit it (e.g. $6^{0} 5^{0} 4^{0} 3^{0} 2^{2} 1^{2}$ and $6^{0} 5^{0} 3^{1} 2^{1} 1^{1}$ denote $2^{2} 1^{2}$ and 321 , respectively).

If rank $f_{2}=2$, then $C_{2}=L+L^{\prime}$, where $L$ and $L^{\prime}$ are two distinct lines. Let $O:=$ $L \cap L^{\prime}$. For $i=1,2,3$, let $m_{i}$ be the number of points $Q$ which is different from the point $O$ with $\left(L . C_{3}\right)_{Q}=i$, and let $n_{i}$ be the number of points $Q^{\prime}$ which is different from the point $O$ with $\left(L^{\prime} \cdot C_{3}\right)_{Q^{\prime}}=i$. Then the notation $3^{m_{3}} 2^{m_{2}} 1^{m_{1}} \cdot 3^{n_{3}} 2^{n_{2}} 1^{n_{1}}$ is called the intersection symbol of $C_{2}=L+L^{\prime}$ and $C_{3}$. We identify the intersection symbol $3^{m_{3}} 2^{m_{2}} 1^{m_{1}} \cdot 3^{n_{3}} 2^{n_{2}} 1^{n_{1}}$ with $3^{n_{3}} 2^{n_{2}} 1^{n_{1}} \cdot 3^{m_{3}} 2^{m_{2}} 1^{m_{1}}$ by changing the roles of $L$ and $L^{\prime}$.

If rank $f_{2}=1$, then $C_{2}=2 L$, where $L$ is a line. For $i=2,4,6$, let $m_{i}$ be the number of points $Q$ with $\left(C_{2} \cdot C_{3}\right)_{Q}=i$. Similarly the notation $\left\langle 6^{m_{6}} 4^{m_{4}} 2^{m_{2}}\right\rangle$ is called the intersection symbol of $C_{2}=2 L$ and $C_{3}$.

By an approach which is different from the one of [2], we will prove that the singularities on $X$ are characterized by the intersection symbol of $C_{2}$ and $C_{3}$ in section 2 .

1.2. The construction of the minimal resolution of singularities of $X$. For a normal singular cubic surface $X$ defined by $(\downarrow)$ with rank $f_{2}>0$, we will construct the minimal resolution of singularities of $X$. We can define a rational map $\Phi$ by

$$
\Phi: \mathbf{P}^{2} \ni\left(y_{0}: y_{1}: y_{2}\right) \mapsto\left(y_{0} f_{2}\left(y_{i}\right): y_{1} f_{2}\left(y_{i}\right): y_{2} f_{2}\left(y_{i}\right): f_{3}\left(y_{i}\right)\right) \in X .
$$

Clearly, $\Phi$ is a birational map, so $X$ is rational. In fact, $\Phi$ is the inverse of a projection of $X$ from the singular point $(0: 0: 0: 1)$ of $X$ to the hyperplane $\left\{x_{3}=0\right\}$.

From the above, a normal singular cubic surface is rational if and only if it is projectively equivalent to a surface defined by $(\downarrow)$ with rank $f_{2}>0$. 
Any point of $C_{2} \cap C_{3}$ is a fundamental point of $\Phi$. Indeed, the birational morphism obtained from the elimination of indeterminacy of $\Phi$ gives the minimal resolution of singularities of $X$. Before showing the above, we recall the language of infinitely near points, which is convenient to describe the position of fundamental points of $\Phi$.

Definition 1.3. Let $S$ be a smooth surface. Then any point on any surface $S^{\prime}$, obtained from $S$ by a finite succession of blowings-up $g: S^{\prime} \rightarrow S$ at points, is called an infinitely near point of $S$. If $Q \in S^{\prime}$ is a point in the open set where $g$ is an isomorphism, then we identify $Q$ with $g(Q)$ as infinitely near points of $S$.

Let $f: \tilde{S} \rightarrow S$ be the blowing-up at a point $P \in S, E$ the exceptional curve, and $C$ a curve on $S$. Then the points of $\tilde{C} \cap E$ are called infinitely near points of $P$ on $C$ of the first order, where $\tilde{C}$ is the strict transform of $C$ by $f$. (Note that if the curve $C$ is smooth at $P$, then $\tilde{C} \cap E$ is a point.) Inductively, for all $j \geqq 2$, infinitely near points of $P$ on $C$ of the $j$-th order are infinitely near points of $Q$ on $\tilde{C}$ of the $(j-1)$-th order, where $Q$ is an infinitely near point of $P$ on $C$ of the first order (see e.g. [1], 8]).

We eliminate fundamental points of $\Phi$ by blowings-up. Let $\Lambda_{0}$ be the linear system on $S_{0}:=\mathbf{P}^{2}$ associated to $\Phi$. Then $\Lambda_{0}$ is contained in the complete linear system $\left|3 E_{0}\right|$, where $E_{0}$ is a line on $\mathbf{P}^{2}$. Let $P_{1} \in S_{0}$ be a base point of $\Lambda_{0}$, and $\varepsilon_{1}: S_{1} \rightarrow S_{0}$ the blowing-up at $P_{1}$. Then the exceptional curve $E_{1}:=\varepsilon_{1}^{-1}\left(P_{1}\right)$ occurs in the fixed part of the linear system $\varepsilon_{1}^{*} \Lambda_{0}$ with some multiplicity. In other words, the linear system $\Lambda_{1}:=\varepsilon_{1}^{*} \Lambda_{0}-k_{1} E_{1}$ has no fixed component, where $k_{1}$ is a minimum value of $\mu_{P_{1}}(C)$ for any $C \in \Lambda_{0}$. Inductively, for $i \geqq 2$, let $P_{i}$ be a base point of $\Lambda_{i-1}, \varepsilon_{i}: S_{i} \rightarrow S_{i-1}$ the blowing-up at $P_{i}$, and $E_{i}:=\varepsilon_{i}^{-1}\left(P_{i}\right)$ the exceptional curve. Similarly we define a linear system on $S_{i}$ by $\Lambda_{i}:=\varepsilon_{i}^{*} \Lambda_{i-1}-k_{i} E_{i}$, where $k_{i}$ is a minimum value of $\mu_{P_{i}}(C)$ for any $C \in \Lambda_{i-1}$. If $n$ is a minimum value that $\Lambda_{n}$ does not have base points, then the $P_{i}$ 's $(1 \leqq i \leqq n)$ are all the fundamental points of $\Phi$.

To determine $n, k_{i}$, and to describe the positions of fundamental points $P_{i}$ according to the intersection symbol, we prepare the next lemma.

Lemma 1.4. Let $S$ be a smooth surface, and $C, C^{\prime}$ effective divisors on $S$, where $C$ and $C^{\prime}$ intersect but have no common divisors. Let $\varepsilon: \tilde{S} \rightarrow S$ be the blowing-up at a point $Q \in C \cap C^{\prime}$, and $E$ the exceptional curve. Assume that $C$ is smooth at Q. Then, we have

(1) $E$ is the only common divisor of $\varepsilon^{*} C$ and $\varepsilon^{*} C^{\prime}$;

(2) let $F:=\varepsilon^{*} C-E$ (i.e., strict transform $\left.\tilde{C}\right), F^{\prime}:=\varepsilon^{*} C^{\prime}-E$; then:

(a) if $\left(C . C^{\prime}\right)_{Q}=1$, then $F$ does not meet $F^{\prime}$ on $E$;

(b) if $\left(C . C^{\prime}\right)_{Q} \geqq 2$, then the only $Q^{\prime}=F \cap E$ is an intersection of $F$ and $F^{\prime}$ on $E$, and $\left(F \cdot F^{\prime}\right)_{Q^{\prime}}=\left(C \cdot C^{\prime}\right)_{Q}-1$.

Proof. (1) is clear, so we prove (2). If $F$ meets $F^{\prime}$ on the exceptional curve $E$, then the intersection of $F$ and $F^{\prime}$ on $E$ must be a point $Q^{\prime}=F \cap E$, since $C$ is smooth at $Q$. Clearly, $F \cdot F^{\prime}=C \cdot C^{\prime}-1$, and the blowing-up at the point $Q$ preserves the intersection multiplicity of $C$ and $C^{\prime}$ at each point $Q^{\prime \prime} \neq Q$, so we have $\left(F . F^{\prime}\right)_{Q^{\prime}}=\left(C . C^{\prime}\right)_{Q}-1$.

If $\left(C \cdot C^{\prime}\right)_{Q}=1$, then $\left(F \cdot F^{\prime}\right)_{Q^{\prime}}=0$, so $F$ does not meet $F^{\prime}$ on $E$. On the other hand, if $\left(C . C^{\prime}\right)_{Q} \geqq 2$, then $F$ meets $F^{\prime}$ on $E$ at the point $Q^{\prime}$ with $\left(F . F^{\prime}\right)_{Q^{\prime}}=$ $\left(C . C^{\prime}\right)_{Q}-1$. 
Since $X$ has only isolated singularities, we have Sing $C_{2} \cap \operatorname{Sing} C_{3}=\emptyset$, where Sing $C_{i}$ consists of points $Q$ in $C_{i}$ with $\mu_{Q}\left(C_{i}\right) \geqq 2$. From Lemma 1.4 and Bezout's theorem, we have the following proposition for $k_{i}, n$ and the positions of fundamental points of $\Phi$.

Proposition 1.5. With the notation and the conditions as above, we have $k_{i}=1$ for $1 \leqq i \leqq 6$, and $n=6$. Moreover, the positions of fundamental points of $\Phi$ are as stated below:

(1) In the case of rank $f_{2}=3$, let $Q_{1} \in C_{2} \cap C_{3}$ with $\left(C_{2} \cdot C_{3}\right)_{Q_{1}}=m$ and $Q_{i}$ be an infinitely near point of $Q_{1}$ on $C_{2}$ of the $(i-1)$-th order for $i=2, \ldots, m$. Then $Q_{1}, \ldots, Q_{m}$ are fundamental points of $\Phi$.

(2) In the case of rank $f_{2}=2$, let $C_{2}=L \cap L^{\prime}$ and $O=L \cap L^{\prime}$. If $Q_{1} \in C_{2} \cap C_{3}$ is not $O$ and $\left(C_{2} . C_{3}\right)_{Q_{1}}=m$, then $Q_{1}, \ldots, Q_{m}$ are fundamental points of $\Phi$, where $Q_{i}(i=2, \ldots, m)$ is an infinitely near point of $Q_{1}$ on $C_{2}$ of the $(i-1)$-th order. If $Q_{1}$ is $O$ and $\left(C_{2} . C_{3}\right)_{Q_{1}}=m$, then $Q_{1}, \ldots, Q_{m}$ are fundamental points of $\Phi$, where $Q_{i}(i=2, \ldots, m)$ is an infinitely near point of $Q_{1}$ on $C_{3}$ of the $(i-1)$-th order.

(3) In the case of rank $f_{2}=1$, let $Q_{1} \in C_{2} \cap C_{3}$ with $\left(C_{2} . C_{3}\right)_{Q_{1}}=m$ and $Q_{i}$ be an infinitely near point of $Q_{1}$ on $C_{3}$ of the $(i-1)$-th order for $i=2, \ldots, m$. Then $Q_{1}, \ldots, Q_{m}$ are fundamental points of $\Phi$.

Proof. We apply Lemma 1.4 to divisors $C_{2}$ and $C_{3}$ on $\mathbf{P}^{2} . k_{i}=1$ is from Lemma 1.4 (1), and $n=6$ is from Bezout's theorem and Lemma 1.4 (2). In regard to the positions of fundamental points, it follows from Lemma 1.4 (2).

Let $\varepsilon:=\varepsilon_{1} \circ \cdots \circ \varepsilon_{6}$; we have a birational morphism $\mu: S_{6} \rightarrow X$ such that $\mu=\Phi \circ \varepsilon$. Since $\Lambda_{0} \subset\left|3 E_{0}\right|, K_{\mathbf{P}^{2}}=-3 E_{0}$ and $k_{i}=1$, we have $\Lambda_{6} \subset\left|-K_{S_{6}}\right|$. Therefore, we get the following corollary.

Corollary 1.6. With the notation and conditions as above, we have $-\omega_{S_{6}}=$ $\mu^{*} \mathcal{O}_{X}(1)$.

We recall the definition for a rational curve with a negative self-intersection number on a smooth surface.

Definition 1.7. Let $S$ be a smooth surface. If $C$ is a smooth rational curve on $S$ with $C^{2}=-d$ where $d$ is a positive integer, then $C$ is called a $(-d)$-curve.

We have some properties for $\mu: S_{6} \rightarrow X$ as follows.

Proposition 1.8. Let $X$ be a normal singular cubic surface in $\mathbf{P}^{3}$ defined by ( $\bullet$ ) with rank $f_{2}>0$. Then the birational morphism $\mu: S_{6} \rightarrow X$ gives the minimal resolution of singularities of $X$. Moreover, $X$ has only rational double points.

Proof. For a $(-1)$-curve $C$ on $S_{6}$, we have $-K_{S_{6}}$. $C=1$, so $\mu(C)$ is not a point. Therefore, $\mu: S_{6} \rightarrow X$ gives the minimal resolution of singularities. By the adjunction formula, the dualizing sheaf $\omega_{X}^{\circ}$ on $X$ is isomorphic to $\mathcal{O}_{X}(-1)$. By Corollary 1.6. $\mu$ is crepant, i.e., $\mu^{*} K_{X}=K_{S_{6}}$. So $X$ has only rational double singularities (see e.g. 14, etc.).

Corollary 1.9. The image of a (-1)-curve on $S_{6}$ by $\mu$ is a line on $X$, and the one of a (-2)-curve by $\mu$ is a singular point on $X$. Moreover, the strict transform of a line on $X$ by $\mu$ is a $(-1)$-curve on $S_{6}$, and the inverse image of a singular point by $\mu$ is a set of connected (-2)-curves on $S_{6}$. 
Proof. Let $C$ be a $(-1)$-curve on $S_{6}$. We have $\mathcal{O}_{X}(1) \cdot \mu(C)=\left(-K_{S_{6}}\right) \cdot C=1$. So the image of $C$ by $\mu$ is a line. Let $C^{\prime}$ be a (-2)-curve on $S_{6}$. Since $C^{\prime} \cdot K_{S_{6}}=0$ and $\mu$ is the minimal resolution, $\mu\left(C^{\prime}\right)$ is a singular point. The latter part is clear by Proposition 1.8 .

By Proposition 1.5, we see that the six fundamental points of $\Phi$ are in an 'almost general position' in the sense of the definition of [5], in any case. So we have the next corollary.

Corollary 1.10. With the notation as above, the anti-canonical divisor $-K_{\tilde{X}}$ is numerically effective, i.e., $-K_{\tilde{X}}$. $E \geqq 0$ for any effective divisor $E$.

Proof. See Demazure [5], p. 39.

Some parts mentioned in this section were known by D. F. Coray and M. A. Tsfasman [4, obtained by using the results of Bruce and Wall [2].

\section{Classification of nORMal Singular CUbiC surfaces}

Hereafter, we denote $S_{6}$ by $\tilde{X}$. In this section, we will determine all $(-1)$-curves and $(-2)$-curves on $\tilde{X}$, according to intersection symbol. Consequently, we will prove the results of Bruce and Wall (Theorem 1) by a different approach than is used in 2].

2.1. (-1)-curves and $(-2)$-curves on $\tilde{X}$. We use the notation in section 1 Let $\pi_{i}:=\varepsilon_{i+1} \circ \varepsilon_{i+2} \circ \cdots \circ \varepsilon_{6}(0 \leqq i \leqq 5)$ and $\pi_{6}:=\mathrm{id}_{S_{6}}$. From section 1, we have Pic $\tilde{X} \simeq \mathbf{Z}^{7}$, which is generated by divisor classes corresponding to the total transforms $\pi_{i}^{*} E_{i}(0 \leqq i \leqq 6)$.

For a curve $C$ on $\tilde{X}$ which is linearly equivalent to $a \pi_{0}^{*} E_{0}-\sum_{i=1}^{6} b_{i} \pi_{i}^{*} E_{i}$, where $a, b_{i}$ are integers, we have the following equations:

$$
\begin{gathered}
C^{2}=a^{2}-\sum_{i=1}^{6} b_{i}^{2}, \\
C . K_{\tilde{X}}=-3 a+\sum_{i=1}^{6} b_{i} .
\end{gathered}
$$

If $C$ is a (-1)-curve, then $C^{2}=C . K_{\tilde{X}}=-1$. Recalling Schwarz's inequality, we have $0 \leqq a \leqq 2$. Therefore, $C$ is linearly equivalent to one of $e_{i}, f_{i, j}, g_{i}$ below:

$$
\begin{gathered}
e_{i}:=\pi_{i}^{*} E_{i}(i=1, \ldots, 6), \\
f_{i, j}:=\pi_{0}^{*} E_{0}-\pi_{i}^{*} E_{i}-\pi_{j}^{*} E_{j}(1 \leqq i<j \leqq 6), \\
g_{i}:=2 \pi_{0}^{*} E_{0}-\sum_{j \neq i} \pi_{j}^{*} E_{j}(i=1, \ldots, 6) .
\end{gathered}
$$

Similarly, if $C$ is a (-2)-curve, then $C^{2}=-2, C \cdot K_{\tilde{X}}=0$. Considering effectivity of $C$, we have $0 \leqq a \leqq 2$. So $C$ is linearly equivalent to one of $p_{i, j}, q_{i, j, k}, r$ below:

$$
\begin{gathered}
p_{i, j}:=\pi_{i}^{*} E_{i}-\pi_{j}^{*} E_{j}(i, j=1, \ldots, 6, i \neq j), \\
q_{i, j, k}:=\pi_{0}^{*} E_{0}-\pi_{i}^{*} E_{i}-\pi_{j}^{*} E_{j}-\pi_{k}^{*} E_{k}(1 \leqq i<j<k \leqq 6), \\
r:=2 \pi_{0}^{*} E_{0}-\sum_{i=1}^{6} \pi_{i}^{*} E_{i} .
\end{gathered}
$$


The intersection numbers of the divisors above are as follows:

$$
\begin{aligned}
e_{i} \cdot e_{j} & =-\delta_{i, j} \\
e_{i} \cdot f_{j, k} & =\delta_{i, j}+\delta_{i, k} \\
e_{i} \cdot g_{j} & =1-\delta_{i, j} \\
e_{i} \cdot p_{j, k} & =\delta_{i, k}-\delta_{i, j} \\
e_{i} \cdot q_{j, k, l} & =\delta_{i, j}+\delta_{i, k}+\delta_{i, l} \\
e_{i} \cdot r & =1 \\
f_{i, j} \cdot f_{k, l} & =1-\delta_{i, k}-\delta_{i, l}-\delta_{j, k}-\delta_{j, l} \\
f_{i, j} \cdot g_{k} & =\delta_{i, k}+\delta_{j, k} \\
f_{i, j} \cdot p_{k, l} & =\delta_{i, k}-\delta_{i, l}+\delta_{j, k}-\delta_{j, l}, \\
f_{i, j} \cdot q_{k, l, m} & =1-\delta_{i, k}-\delta_{i, l}-\delta_{i, m}-\delta_{j, k}-\delta_{j, l}-\delta_{j, m}, \\
f_{i, j} \cdot r & =0 \\
g_{i} \cdot g_{j} & =-\delta_{i, j}, \\
g_{i} \cdot p_{j, k} & =\delta_{i, k}-\delta_{i, j}, \\
g_{i} \cdot q_{j, k, l} & =\delta_{i, j}+\delta_{i, k}+\delta_{i, l}-1, \\
g_{i} \cdot r & =-1 \\
p_{i, j} \cdot p_{k, l} & =\delta_{i, l}+\delta_{j, k}-\delta_{i, k}-\delta_{j, l}, \\
p_{i, j} \cdot q_{k, l, m} & =\delta_{i, k}+\delta_{i, l}+\delta_{i, m}-\delta_{j, k}-\delta_{j, l}-\delta_{j, m} \\
p_{i, j} \cdot r & =0 \\
q_{i, j, k} \cdot q_{l, m, n} & =1-\delta_{i, l}-\delta_{i, m}-\delta_{i, n}-\delta_{j, l}-\delta_{j, m}-\delta_{j, n}-\delta_{k, l}-\delta_{k, m}-\delta_{k, n}, \\
q_{i, j, k} \cdot r & =-1 \\
r^{2} & =-2
\end{aligned}
$$

where $\delta_{i, j}$ is a Kronecker delta.

Definition 2.1. We denote by $M_{\tilde{X}}$ (resp. $N_{\tilde{X}}$ ) the set of $e_{i}$ 's, $f_{i, j}$ 's and $g_{i}$ 's (resp. $p_{i, j}$ 's, $q_{i, j, k}$ 's and $r$ ). We denote by $I_{\tilde{X}}$ (resp. $R_{\tilde{X}}$ ) the set of all $(-1)$-curves (resp. all (-2)-curves) on $\tilde{X}$.

We recall well-known facts about the irreducibility of divisors on a smooth surface.

Lemma 2.2. Let $S$ be a smooth surface and let $D$ be an effective divisor on $S$. If $D . C<0$ for a prime divisor $C$, then $D=C+E$, where $E$ is an effective divisor.

Proof. It follows from the fact that the intersection number of two effective divisors without common divisors is positive or zero.

Lemma 2.3. Let $S$ be a smooth surface and let $C$ be an effective divisor on $S$ with $C^{2}<0$. If $C$ is a prime divisor, then the complete linear system $|C|$ consists of a unique element $C$. On the other hand, if $C$ is not a prime divisor, then any member of $|C|$ is not a prime divisor.

Proof. Let $C$ be a prime divisor. For an effective divisor $E \in|C|$, we have $E . C=$ $C^{2}<0$. By Lemma 2.2, we have $E=C+F$, where $F$ is effective. So $F$ is linearly equivalent to a zero divisor. If $F$ is not a zero divisor, then $A . F>0$ for an ample divisor $A$ by the Nakai-Moishezon Criterion, so it is a contradiction. Hence, $F=0$ and $E=C$. The latter part is clear from the first part. 
For any $C \in I_{\tilde{X}}\left(\right.$ resp. $\left.R_{\tilde{X}}\right)$, there exists a unique divisor $D \in M_{\tilde{X}}\left(\right.$ resp. $\left.N_{\tilde{X}}\right)$ with $C \sim D$, since any distinct two divisors of $M_{\tilde{X}}$ (resp. $N_{\tilde{X}}$ ) are not linearly equivalent. So we can define maps $\varphi_{I}: I_{\tilde{X}} \rightarrow M_{\tilde{X}}$ and $\varphi_{R}: R_{\tilde{X}} \rightarrow N_{\tilde{X}}$, which are injective. Using the following proposition, we can determine $I_{\tilde{X}}$ and $R_{\tilde{X}}$ according to the intersection symbol by computing intersection numbers. Furthermore we can get the configuration of $(-1)$-curves and $(-2)$-curves on $\tilde{X}$ by (困).

Proposition 2.4. With the notation above, let $I^{\prime}$ be a subset of $I_{\tilde{X}}$ and let $R^{\prime}$ be a subset of $R_{\tilde{X}}$. If there exists $C \in I^{\prime} \cup R^{\prime}$ with $D . C<0$ for any $D \in M_{\tilde{X}} \backslash \varphi_{I}\left(I^{\prime}\right)$, then $I_{\tilde{X}}=I^{\prime}$. Similarly, if there exists $C \in I^{\prime} \cup R^{\prime}$ with D.C $<0$ for any $D \in$ $N_{\tilde{X}} \backslash \varphi_{R}\left(R^{\prime}\right)$, then $R_{\tilde{X}}=R^{\prime}$.

Proof. We prove the first part. We assume that $I^{\prime} \varsubsetneqq I_{\tilde{X}}$. For any curve $C^{\prime} \in I_{\tilde{X}} \backslash I^{\prime}$, $D:=\varphi_{I}\left(C^{\prime}\right)$ is not in $\varphi_{I}\left(I^{\prime}\right)$. From the conditions, there exists $C \in I^{\prime} \cup R^{\prime}$ with $D . C<0$, so we have $C^{\prime} . C<0$. Since $C^{\prime}$ is effective, there exists an effective divisor $E$ such that $C^{\prime}=C+E$. On the other hand, $C^{\prime}$ is a prime divisor, so $E$ is zero. Therefore, we have $C^{\prime}=C$, a contradiction. Hence, $I^{\prime}=I_{\tilde{X}}$. The latter part is similar.

We prepare the notation to describe curves on $\tilde{X}$.

Definition 2.5. We denote the strict transform of a curve $C \subset S_{i}$ via the morphism $\pi_{i}: \tilde{X} \rightarrow S_{i}$ by $\tilde{C} \subset \tilde{X}$, for short. For the fundamental points $P_{i}$ of $\Phi$, let $\wp:=\left\{P_{i}(1 \leqq i \leqq 6)\right\}$, and $\wp^{\prime}:=\left\{P_{i_{1}}, \ldots P_{i_{m}}\right\} \subset \wp$. If a line on $\mathbf{P}^{2}$ (or the strict transform on $S_{i}$ ) passes through all points of $\wp^{\prime}$ but does not pass through all points of $\wp \backslash \wp^{\prime}$, then we denote the line by $L_{i_{1}, \ldots, i_{m}}$. We note that the strict transform $\tilde{L}_{i_{1}, \ldots, i_{m}}$ is linearly equivalent to $\pi_{0}^{*} E_{0}-\sum_{j=1}^{m} \pi_{i_{j}}^{*} E_{i_{j}}$.

Hereafter, we determine $I_{\tilde{X}}$ and $R_{\tilde{X}}$ by using Proposition 2.4 with respect to each intersection symbol.

2.2. The case of rank $f_{2}=3$. Then $\tilde{C}_{2}$ is always a $(-2)$-curve, and there are no $(-2)$-curves which meet with $\tilde{C}_{2}$, so $X$ has an $A_{1}$ singularity in this case.

2.2.1. Type $1^{6}$. In the case of the intersection symbol $1^{6}$, we determine the $I_{\tilde{X}}, R_{\tilde{X}}$ by using Proposition 2.4. Then the fundamental points $P_{i}$ are intersection points $C_{2} \cap C_{3}$. We can choose $I^{\prime} \subset I_{\tilde{X}}$ and $R^{\prime} \subset R_{\tilde{X}}$ as follows:

$$
I^{\prime}=\left\{\tilde{E}_{i}(1 \leqq i \leqq 6), \tilde{L}_{i, j}(1 \leqq i<j \leqq 6)\right\}, R^{\prime}=\left\{\tilde{C}_{2}\right\} .
$$

Then, we have

$$
\begin{gathered}
M_{\tilde{X}} \backslash \varphi_{I}\left(I^{\prime}\right)=\left\{g_{i}(1 \leqq i \leqq 6)\right\}, \\
N_{\tilde{X}} \backslash \varphi_{R}\left(R^{\prime}\right)=\left\{p_{i, j}(1 \leqq i, j \leqq 6, i \neq j), q_{i, j, k}(1 \leqq i<j<k \leqq 6)\right\} .
\end{gathered}
$$

For $g_{i} \in M_{\tilde{X}} \backslash \varphi_{I}\left(I^{\prime}\right)$ and $\tilde{C}_{2} \in R^{\prime}$, we have $g_{i} . \tilde{C}_{2}=g_{i} . r=-1<0$. Therefore, we have $I_{\tilde{X}}=I^{\prime}$ by Proposition 2.4. Similarly, for $p_{i, j}(1 \leqq i, j \leqq 6, i \neq j)$ and $\tilde{E}_{i} \in I^{\prime}$, we have $p_{i, j} \cdot \tilde{E}_{i}=p_{i, j} \cdot e_{i}=-1<0$. For $q_{i, j, k}(1 \leqq i<j<k \leqq 6)$ and $\tilde{C}_{2} \in R^{\prime}$, we have $q_{i, j, k} \cdot \tilde{C}_{2}=q_{i, j, k} . r=-1<0$. Therefore, we have $R_{\tilde{X}}=R^{\prime}$ by Proposition 2.4. In particular, $X$ has the only $A_{1}$ singularity, and $X$ contains exactly 21 lines by Corollary 1.9 .

The calculation above is very simple, but we generally need many of them. Actually, for any $D \in\left(M_{\tilde{X}} \cup N_{\tilde{X}}\right) \backslash\left(\varphi_{I}\left(I^{\prime}\right) \cup \varphi_{R}\left(R^{\prime}\right)\right)$, we verify that there exists 
$C \in \varphi_{I}\left(I^{\prime}\right) \cup \varphi_{R}\left(R^{\prime}\right)$ with $D . C<0$ by using (四) with respect to each intersection symbol. Hereafter, only the results are recorded.

2.2.2. Type $21^{4}$. Let $P_{1}$ be a point of $C_{2} \cap C_{3}$ with $\left(C_{2} . C_{3}\right)_{P_{1}}=2, P_{2}$ an infinitely near point of $P_{1}$ on $C_{2}$ of the first order, and $P_{i}(i=3,4,5,6)$ a point of $C_{2} \cap C_{3}$ with $\left(C_{2} . C_{3}\right)_{P_{i}}=1$. Then,

$$
\begin{gathered}
I_{\tilde{X}}=\left\{\tilde{E}_{i}(2 \leqq i \leqq 6), \quad \tilde{L}_{i, j}(i, j \in\{1,3,4,5,6\}, i<j), \tilde{L}_{1,2}\right\}, \\
R_{\tilde{X}}=\left\{\tilde{C}_{2}, \tilde{E}_{1}\right\} .
\end{gathered}
$$

Since $\tilde{C}_{2} \sim r, \tilde{E}_{1} \sim p_{1,2}$ and $r \cdot p_{1,2}=0, X$ has only $2 A_{1}$ singularities and contains exactly 16 lines.

2.2.3. Type $31^{3}$. Let $P_{1}$ be a point of $C_{2} \cap C_{3}$ with $\left(C_{2} \cdot C_{3}\right)_{P_{1}}=3, P_{i}(i=2,3)$ an infinitely near point of $P_{1}$ on $C_{2}$ of the $(i-1)$-th order, and $P_{i}(i=4,5,6)$ a point of $C_{2} \cap C_{3}$ with $\left(C_{2} \cdot C_{3}\right)_{P_{i}}=1$. Then,

$$
\begin{gathered}
I_{\tilde{X}}=\left\{\tilde{E}_{3}, \tilde{E}_{4}, \tilde{E}_{5}, \tilde{E}_{6}, \tilde{L}_{i, j}(i, j \in\{1,4,5,6\}, i<j), \tilde{L}_{1,2}\right\}, \\
R_{\tilde{X}}=\left\{\tilde{C}_{2}, \tilde{E}_{1}, \tilde{E}_{2}\right\},
\end{gathered}
$$

so $X$ has only $A_{1} A_{2}$ singularities and contains exactly 11 lines.

2.2.4. Type $2^{2} 1^{2}$. Let $P_{1}, P_{3}$ be points of $C_{2} \cap C_{3}$ with $\left(C_{2} . C_{3}\right)_{P_{i}}=2, P_{i}(i=2,4)$ an infinitely near point of $P_{i-1}$ on $C_{2}$ of the first order, and let $P_{5}, P_{6}$ be points of $C_{2} \cap C_{3}$ with $\left(C_{2} . C_{3}\right)_{P_{i}}=1$. Then,

$$
\begin{gathered}
I_{\tilde{X}}=\left\{\tilde{E}_{2}, \tilde{E}_{4}, \tilde{E}_{5}, \tilde{E}_{6}, \tilde{L}_{i, j}(i, j \in\{1,3,5,6\}, i<j), \tilde{L}_{1,2}, \tilde{L}_{3,4}\right\}, \\
R_{\tilde{X}}=\left\{\tilde{C}_{2}, \tilde{E}_{1}, \tilde{E}_{3}\right\},
\end{gathered}
$$

so $X$ has only $3 A_{1}$ singularities and contains exactly 12 lines.

2.2.5. Type $41^{2}$. Let $P_{1}$ be a point of $C_{2} \cap C_{3}$ with $\left(C_{2} . C_{3}\right)_{P_{1}}=4, P_{i}(i=2,3,4)$ an infinitely near point of $P_{1}$ on $C_{2}$ of the $(i-1)$-th order, and $P_{5}, P_{6}$ be points of $C_{2} \cap C_{3}$ with $\left(C_{2} . C_{3}\right)_{P_{i}}=1$. Then,

$$
\begin{gathered}
I_{\tilde{X}}=\left\{\tilde{E}_{4}, \tilde{E}_{5}, \tilde{E}_{6}, \tilde{L}_{1,5}, \tilde{L}_{1,6}, \tilde{L}_{5,6}, \tilde{L}_{1,2}\right\}, \\
R_{\tilde{X}}=\left\{\tilde{C}_{2}, \tilde{E}_{1}, \tilde{E}_{2}, \tilde{E}_{3}\right\},
\end{gathered}
$$

so $X$ has only $A_{1} A_{3}$ singularities and contains exactly 7 lines.

2.2.6. Type 321. Let $P_{1}$ be a point of $C_{2} \cap C_{3}$ with $\left(C_{2} . C_{3}\right)_{P_{1}}=3, P_{i}(i=2,3)$ an infinitely near point of $P_{1}$ on $C_{2}$ of the $(i-1)$-th order, $P_{4}$ a point of $C_{2} \cap C_{3}$ with $\left(C_{2} . C_{3}\right)_{P_{4}}=2, P_{5}$ an infinitely near point of $P_{4}$ on $C_{2}$ of the first order, and $P_{6}$ a point of $C_{2} \cap C_{3}$ with $\left(C_{2} \cdot C_{3}\right)_{P_{6}}=1$. Then,

$$
\begin{gathered}
I_{\tilde{X}}=\left\{\tilde{E}_{3}, \tilde{E}_{5}, \tilde{E}_{6}, \tilde{L}_{1,4}, \tilde{L}_{1,6}, \tilde{L}_{4,6}, \tilde{L}_{1,2}, \tilde{L}_{4,5}\right\}, \\
R_{\tilde{X}}=\left\{\tilde{C}_{2}, \tilde{E}_{1}, \tilde{E}_{2}, \tilde{E}_{4}\right\},
\end{gathered}
$$

so $X$ has only $2 A_{1} A_{2}$ singularities and contains exactly 8 lines. 
2.2.7. Type $2^{3}$. Let $P_{1}, P_{3}, P_{5}$ be points of $C_{2} \cap C_{3}$ with $\left(C_{2} . C_{3}\right)_{P_{i}}=2$, and $P_{i}(i=$ $2,4,6)$ an infinitely near point of $P_{i-1}$ on $C_{2}$ of the first order. Then,

$$
\begin{gathered}
I_{\tilde{X}}=\left\{\tilde{E}_{2}, \tilde{E}_{4}, \tilde{E}_{6}, \tilde{L}_{1,3}, \tilde{L}_{1,5}, \tilde{L}_{3,5}, \tilde{L}_{1,2}, \tilde{L}_{3,4}, \tilde{L}_{5,6}\right\}, \\
R_{\tilde{X}}=\left\{\tilde{C}_{2}, \tilde{E}_{1}, \tilde{E}_{3}, \tilde{E}_{5}\right\},
\end{gathered}
$$

so $X$ has only $4 A_{1}$ singularities and contains exactly 9 lines.

2.2.8. Type 51. Let $P_{1}$ be a point of $C_{2} \cap C_{3}$ with $\left(C_{2} . C_{3}\right)_{P_{1}}=5, P_{i}(i=2,3,4,5)$ an infinitely near point of $P_{1}$ on $C_{2}$ of the $(i-1)$-th order, and $P_{6}$ a point of $C_{2} \cap C_{3}$ with $\left(C_{2} \cdot C_{3}\right)_{P_{6}}=1$. Then,

$$
I_{\tilde{X}}=\left\{\tilde{E}_{5}, \tilde{E}_{6}, \tilde{L}_{1,6}, \tilde{L}_{1,2}\right\}, R_{\tilde{X}}=\left\{\tilde{C}_{2}, \tilde{E}_{1}, \tilde{E}_{2}, \tilde{E}_{3}, \tilde{E}_{4}\right\},
$$

so $X$ has only $A_{1} A_{4}$ singularities and contains exactly 4 lines.

2.2.9. Type 42. Let $P_{1}$ be a point of $C_{2} \cap C_{3}$ with $\left(C_{2} . C_{3}\right)_{P_{1}}=4, P_{i}(i=2,3,4)$ an infinitely near point of $P_{1}$ on $C_{2}$ of the $(i-1)$-th order, $P_{5}$ a point of $C_{2} \cap C_{3}$ with $\left(C_{2} . C_{3}\right)_{P_{5}}=2$, and $P_{6}$ an infinitely near point of $P_{5}$ on $C_{2}$ of the first order. Then,

$$
I_{\tilde{X}}=\left\{\tilde{E}_{4}, \tilde{E}_{6}, \tilde{L}_{1,5}, \tilde{L}_{1,2}, \tilde{L}_{5,6}\right\}, R_{\tilde{X}}=\left\{\tilde{C}_{2}, \tilde{E}_{1}, \tilde{E}_{2}, \tilde{E}_{3}, \tilde{E}_{5}\right\},
$$

so $X$ has only $2 A_{1} A_{3}$ singularities and contains exactly 5 lines.

2.2.10. Type $3^{2}$. Let $P_{1}, P_{4}$ be points of $C_{2} \cap C_{3}$ with $\left(C_{2} . C_{3}\right)_{P_{i}}=3, P_{i}(i=2,3)$ an infinitely near point of $P_{1}$ on $C_{2}$ of the $(i-1)$-th order, and $P_{i}(i=5,6)$ an infinitely near point of $P_{4}$ on $C_{2}$ of the $(i-4)$-th order. Then,

$$
I_{\tilde{X}}=\left\{\tilde{E}_{3}, \tilde{E}_{6}, \tilde{L}_{1,4}, \tilde{L}_{1,2}, \tilde{L}_{4,5}\right\}, R_{\tilde{X}}=\left\{\tilde{C}_{2}, \tilde{E}_{1}, \tilde{E}_{2}, \tilde{E}_{4}, \tilde{E}_{5}\right\},
$$

so $X$ has only $A_{1} 2 A_{2}$ singularities and contains exactly 5 lines.

2.2.11. Type 6. Let $P_{1}$ be a point of $C_{2} \cap C_{3}$ with $\left(C_{2} . C_{3}\right)_{P_{1}}=6$ and $P_{i}(i=2, \ldots, 6)$ an infinitely near point of $P_{1}$ on $C_{2}$ of the $(i-1)$-th order. Then,

$$
I_{\tilde{X}}=\left\{\tilde{E}_{6}, \tilde{L}_{1,2}\right\}, R_{\tilde{X}}=\left\{\tilde{C}_{2}, \tilde{E}_{1}, \tilde{E}_{2}, \tilde{E}_{3}, \tilde{E}_{4}, \tilde{E}_{5}\right\}
$$

so $X$ has only $A_{1} A_{5}$ singularities and contains exactly 2 lines.

2.3. The case of rank $f_{2}=2$. Let $C_{2}=L+L^{\prime}$ and $O=L \cap L^{\prime}$, where $L, L^{\prime}$ are lines.

2.3.1. Type $1^{3} \cdot 1^{3}$. Let $P_{1}, P_{2}, P_{3}$ be points of $C_{3} \cap L$ with $\left(L . C_{3}\right)_{P_{i}}=1$, and $P_{4}, P_{5}, P_{6}$ be points of $C_{3} \cap L^{\prime}$ with $\left(L^{\prime} . C_{3}\right)_{P_{i}}=1$. Then,

$$
\begin{gathered}
I_{\tilde{X}}=\left\{\tilde{E}_{i}(1 \leqq i \leqq 6), \tilde{L}_{i, j}(i \in\{1,2,3\}, j \in\{4,5,6\})\right\}, \\
R_{\tilde{X}}=\left\{\tilde{L}=\tilde{L}_{1,2,3}, \tilde{L}^{\prime}=\tilde{L}_{4,5,6}\right\} .
\end{gathered}
$$

Since $\tilde{L} \sim q_{1,2,3}, \tilde{L}^{\prime} \sim q_{4,5,6}$ and $q_{1,2,3} . q_{4,5,6}=1, X$ has the only $A_{2}$ singularity and $X$ contains exactly 15 lines.

2.3.2. Type $1^{3} .21$. Let $P_{1}, P_{2}, P_{3}$ be points of $C_{3} \cap L$ with $\left(L . C_{3}\right)_{P_{i}}=1, P_{4}$ a point of $C_{3} \cap L^{\prime}$ with $\left(L^{\prime} . C_{3}\right)_{P_{4}}=2, P_{5}$ an infinitely near point of $P_{4}$ on $C_{2}$ of the first order, and $P_{6}$ a point of $C_{3} \cap L^{\prime}$ with $\left(L^{\prime} \cdot C_{3}\right)_{P_{6}}=1$. Then,

$$
\begin{gathered}
I_{\tilde{X}}=\left\{\tilde{E}_{i}(i=1,2,3,5,6), \tilde{L}_{i, j}(i \in\{1,2,3\}, j \in\{4,6\})\right\}, \\
R_{\tilde{X}}=\left\{\tilde{L}=\tilde{L}_{1,2,3}, \tilde{L}^{\prime}=\tilde{L}_{4,5,6}, \tilde{E}_{4}\right\},
\end{gathered}
$$

so $X$ has only $A_{2} A_{1}$ singularities and contains exactly 11 lines. 
2.3.3. Type $1^{3}$.3. Let $P_{1}, P_{2}, P_{3}$ be points of $C_{3} \cap L$ with $\left(L . C_{3}\right)_{P_{i}}=1, P_{4}$ a point of $C_{3} \cap L^{\prime}$ with $\left(L^{\prime} . C_{3}\right)_{P_{4}}=3$, and $P_{i}(i=5,6)$ an infinitely near point of $P_{4}$ on $C_{2}$ of the $(i-4)$-th order. Then,

$$
\begin{aligned}
I_{\tilde{X}} & =\left\{\tilde{E}_{1}, \tilde{E}_{2}, \tilde{E}_{3}, \tilde{E}_{6}, \tilde{L}_{1,4}, \tilde{L}_{2,4}, \tilde{L}_{3,4}\right\}, \\
R_{\tilde{X}} & =\left\{\tilde{L}=\tilde{L}_{1,2,3}, \tilde{L}^{\prime}=\tilde{L}_{4,5,6}, \tilde{E}_{4}, \tilde{E}_{5}\right\},
\end{aligned}
$$

so $X$ has only $2 A_{2}$ singularities and contains exactly 7 lines.

2.3.4. Type 21.21. Let $P_{1}$ be a point of $C_{3} \cap L$ with $\left(L . C_{3}\right)_{P_{1}}=2, P_{2}$ an infinitely near point of $P_{1}$ on $C_{2}$ of the first order, $P_{3}$ a point of $C_{3} \cap L$ with $\left(L . C_{3}\right)_{P_{3}}=1$, $P_{4}$ a point of $C_{3} \cap L^{\prime}$ with $\left(L^{\prime} . C_{3}\right)_{P_{4}}=2, P_{5}$ an infinitely near point of $P_{4}$ on $C_{2}$ of the first order, and $P_{6}$ a point of $C_{3} \cap L^{\prime}$ with $\left(L^{\prime} . C_{3}\right)_{P_{6}}=1$. Then,

$$
\begin{gathered}
I_{\tilde{X}}=\left\{\tilde{E}_{2}, \tilde{E}_{3}, \tilde{E}_{5}, \tilde{E}_{6}, \tilde{L}_{1,4}, \tilde{L}_{1,6}, \tilde{L}_{3,4}, \tilde{L}_{3,6}\right\}, \\
R_{\tilde{X}}=\left\{\tilde{L}=\tilde{L}_{1,2,3}, \tilde{L}^{\prime}=\tilde{L}_{4,5,6}, \tilde{E}_{1}, \tilde{E}_{4}\right\},
\end{gathered}
$$

so $X$ has only $A_{2} 2 A_{1}$ singularities and contains exactly 8 lines.

2.3.5. Type 21.3. Let $P_{1}$ be a point of $C_{3} \cap L$ with $\left(L . C_{3}\right)_{P_{1}}=2, P_{2}$ an infinitely near point of $P_{1}$ on $C_{2}$ of the first order, $P_{3}$ a point of $C_{3} \cap L$ with $\left(L . C_{3}\right)_{P_{3}}=1$, $P_{4}$ a point of $C_{3} \cap L^{\prime}$ with $\left(L^{\prime} \cdot C_{3}\right)_{P_{4}}=3$, and $P_{i}(i=5,6)$ an infinitely near point of $P_{4}$ on $C_{2}$ of the $(i-4)$-th order. Then,

$$
\begin{gathered}
I_{\tilde{X}}=\left\{\tilde{E}_{2}, \tilde{E}_{3}, \tilde{E}_{6}, \tilde{L}_{1,4}, \tilde{L}_{3,4}\right\}, \\
R_{\tilde{X}}=\left\{\tilde{L}=\tilde{L}_{1,2,3}, \tilde{L}^{\prime}=\tilde{L}_{4,5,6}, \tilde{E}_{1}, \tilde{E}_{4}, \tilde{E}_{5}\right\},
\end{gathered}
$$

so $X$ has only $2 A_{2} A_{1}$ singularities and contains exactly 5 lines.

2.3.6. Type 3.3. Let $P_{1}$ be a point of $C_{3} \cap L$ with $\left(L . C_{3}\right)_{P_{1}}=3, P_{i}(i=2,3)$ an infinitely near point of $P_{1}$ on $C_{2}$ of the $(i-1)$-th order, $P_{4}$ a point of $C_{3} \cap L^{\prime}$ with $\left(L^{\prime} . C_{3}\right)_{P_{4}}=3$, and $P_{i}(i=5,6)$ an infinitely near point of $P_{4}$ on $C_{2}$ of the $(i-4)$-th order. Then,

$$
\begin{gathered}
I_{\tilde{X}}=\left\{\tilde{E}_{3}, \tilde{E}_{6}, \tilde{L}_{1,4}\right\}, \\
R_{\tilde{X}}=\left\{\tilde{L}=\tilde{L}_{1,2,3}, \tilde{L}^{\prime}=\tilde{L}_{4,5,6}, \tilde{E}_{1}, \tilde{E}_{2}, \tilde{E}_{4}, \tilde{E}_{5}\right\},
\end{gathered}
$$

so $X$ has only $3 A_{2}$ singularities and contains exactly 3 lines.

2.3.7. Type $1^{2} .1^{2}$. Let $P_{1}, P_{2} \neq O$ be points of $C_{3} \cap L$ with $\left(L . C_{3}\right)_{P_{i}}=1, P_{3}, P_{4} \neq O$ points of $C_{3} \cap L^{\prime}$ with $\left(L^{\prime} . C_{3}\right)_{P_{i}}=1, P_{5}=O$, and let $P_{6}$ be an infinitely near point of $P_{5}$ on $C_{3}$ of the first order. Then,

$$
\begin{gathered}
I_{\tilde{X}}=\left\{\tilde{E}_{i}(i \in\{1,2,3,4,6\}), \tilde{L}_{1,3}, \tilde{L}_{1,4}, \tilde{L}_{2,3}, \tilde{L}_{2,4}, \tilde{L}_{5,6}\right\}, \\
R_{\tilde{X}}=\left\{\tilde{L}=\tilde{L}_{1,2,5}, \tilde{L}^{\prime}=\tilde{L}_{3,4,5}, \tilde{E}_{5}\right\},
\end{gathered}
$$

so $X$ has the only $A_{3}$ singularity and contains exactly 10 lines. 
2.3.8. Type $1^{2}$.2. Let $P_{1}, P_{2} \neq O$ be points of $C_{3} \cap L$ with $\left(L . C_{3}\right)_{P_{i}}=1, P_{3} \neq O$ a point of $C_{3} \cap L^{\prime}$ with $\left(L^{\prime} . C_{3}\right)_{P_{3}}=2, P_{4}$ an infinitely near point of $P_{3}$ on $C_{2}$ of the first order, $P_{5}=O$, and $P_{6}$ an infinitely near point of $P_{5}$ on $C_{3}$ of the first order. Then,

$$
\begin{aligned}
I_{\tilde{X}} & =\left\{\tilde{E}_{1}, \tilde{E}_{2}, \tilde{E}_{4}, \tilde{E}_{6}, \tilde{L}_{1,3}, \tilde{L}_{2,3}, \tilde{L}_{5,6}\right\}, \\
R_{\tilde{X}} & =\left\{\tilde{L}=\tilde{L}_{1,2,5}, \tilde{L}^{\prime}=\tilde{L}_{3,4,5}, \tilde{E}_{3}, \tilde{E}_{5}\right\},
\end{aligned}
$$

so $X$ has only $A_{3} A_{1}$ singularities and contains exactly 7 lines.

2.3.9. Type 2.2. Let $P_{1} \neq O$ be a point of $C_{3} \cap L$ with $\left(L . C_{3}\right)_{P_{1}}=2, P_{2}$ an infinitely near point of $P_{1}$ on $C_{2}$ of the first order, $P_{3} \neq O$ a point of $C_{3} \cap L^{\prime}$ with $\left(L^{\prime} . C_{3}\right)_{P_{3}}=2, P_{4}$ an infinitely near point of $P_{3}$ on $C_{2}$ of the first order, $P_{5}=O$, and $P_{6}$ an infinitely near point of $P_{5}$ on $C_{3}$ of the first order. Then,

$$
\begin{gathered}
I_{\tilde{X}}=\left\{\tilde{E}_{2}, \tilde{E}_{4}, \tilde{E}_{6}, \tilde{L}_{1,3}, \tilde{L}_{5,6}\right\}, \\
R_{\tilde{X}}=\left\{\tilde{L}=\tilde{L}_{1,2,5}, \tilde{L}^{\prime}=\tilde{L}_{3,4,5}, \tilde{E}_{1}, \tilde{E}_{3}, \tilde{E}_{5}\right\},
\end{gathered}
$$

so $X$ has only $A_{3} 2 A_{1}$ singularities and contains exactly 5 lines.

2.3.10. Type $1^{2}$.1. Let $P_{1}, P_{2} \neq O$ be points of $C_{3} \cap L$ with $\left(L . C_{3}\right)_{P_{i}}=1, P_{3} \neq O$ a point of $C_{3} \cap L^{\prime}$ with $\left(L^{\prime} . C_{3}\right)_{P_{3}}=1, P_{4}=O$, and $P_{i}(i=5,6)$ an infinitely near point of $P_{4}$ on $C_{3}$ of the $(i-4)$-th order. Then,

$$
\begin{gathered}
I_{\tilde{X}}=\left\{\tilde{E}_{1}, \tilde{E}_{2}, \tilde{E}_{3}, \tilde{E}_{6}, \tilde{L}_{1,3}, \tilde{L}_{2,3}\right\}, \\
R_{\tilde{X}}=\left\{\tilde{L}=\tilde{L}_{1,2,4}, \tilde{L}^{\prime}=\tilde{L}_{3,4,5}, \tilde{E}_{4}, \tilde{E}_{5}\right\},
\end{gathered}
$$

so $X$ has the only $A_{4}$ singularity and contains exactly 6 lines.

2.3.11. Type 2.1. Let $P_{1} \neq O$ be a point of $C_{3} \cap L$ with $\left(L . C_{3}\right)_{P_{1}}=2, P_{2}$ an infinitely near point of $P_{1}$ on $C_{2}$ of the first order, $P_{3} \neq O$ a point of $C_{3} \cap L^{\prime}$ with $\left(L^{\prime} \cdot C_{3}\right)_{P_{3}}=1, P_{4}=O$, and $P_{i}(i=5,6)$ an infinitely near point of $P_{4}$ on $C_{3}$ of the $(i-4)$-th order. Then,

$$
\begin{gathered}
I_{\tilde{X}}=\left\{\tilde{E}_{2}, \tilde{E}_{3}, \tilde{E}_{6}, \tilde{L}_{1,3}\right\}, \\
R_{\tilde{X}}=\left\{\tilde{L}=\tilde{L}_{1,2,4}, \tilde{L}^{\prime}=\tilde{L}_{3,4,5}, \tilde{E}_{1}, \tilde{E}_{4}, \tilde{E}_{5}\right\},
\end{gathered}
$$

so $X$ has only $A_{4} A_{1}$ singularities and contains exactly 4 lines.

2.3.12. Type $1^{2} .0$. Let $P_{1}, P_{2} \neq O$ be points of $C_{3} \cap L$ with $\left(L . C_{3}\right)_{P_{i}}=1, P_{3}=O$, and $P_{i}(i=4,5,6)$ an infinitely near point of $P_{3}$ on $C_{3}$ of the $(i-3)$-th order. Then,

$$
\begin{gathered}
I_{\tilde{X}}=\left\{\tilde{E}_{1}, \tilde{E}_{2}, \tilde{E}_{6}\right\}, \\
R_{\tilde{X}}=\left\{\tilde{L}=\tilde{L}_{1,2,3}, \tilde{L}^{\prime}=\tilde{L}_{3,4,5}, \tilde{E}_{3}, \tilde{E}_{4}, \tilde{E}_{5}\right\},
\end{gathered}
$$

so $X$ has the only $A_{5}$ singularity and contains exactly 3 lines.

2.3.13. Type 2.0. Let $P_{1} \neq O$ be a point of $C_{3} \cap L$ with $\left(L . C_{3}\right)_{P_{1}}=2, P_{2}$ an infinitely near point of $P_{1}$ on $C_{2}$ of the first order, $P_{3}=O$, and $P_{i}(i=4,5,6)$ an infinitely near point of $P_{3}$ on $C_{3}$ of the $(i-3)$-th order. Then,

$$
I_{\tilde{X}}=\left\{\tilde{E}_{2}, \tilde{E}_{6}\right\}, R_{\tilde{X}}=\left\{\tilde{L}=\tilde{L}_{1,2,3}, \tilde{L}^{\prime}=\tilde{L}_{3,4,5}, \tilde{E}_{1}, \tilde{E}_{3}, \tilde{E}_{4}, \tilde{E}_{5}\right\}
$$

so $X$ has only $A_{5} A_{1}$ singularities and contains exactly 2 lines.

2.4. The case of rank $f_{2}=1$. We put $C_{2}=2 L$, where $L$ is a line. 
2.4.1. Type $\left\langle 2^{3}\right\rangle$. Let $P_{1}, P_{3}, P_{5}$ be points with $\left(C_{2} \cdot C_{3}\right)_{P_{i}}=2$, and $P_{i}(i=2,4,6)$ an infinitely near point of $P_{i-1}$ on $C_{3}$ of the first order. Then,

$$
\begin{gathered}
I_{\tilde{X}}=\left\{\tilde{E}_{2}, \tilde{E}_{4}, \tilde{E}_{6}, \tilde{L}_{1,2}, \tilde{L}_{3,4}, \tilde{L}_{5,6}\right\}, \\
R_{\tilde{X}}=\left\{\tilde{L}=\tilde{L}_{1,2,3}, \tilde{E}_{1}, \tilde{E}_{3}, \tilde{E}_{5}\right\},
\end{gathered}
$$

so $X$ has the only $D_{4}$ singularity and contains exactly 6 lines.

2.4.2. Type $\langle 42\rangle$. Let $P_{1}$ be a point with $\left(C_{2} . C_{3}\right)_{P_{1}}=4, P_{i}(i=2,3,4)$ an infinitely near point of $P_{1}$ on $C_{3}$ of the $(i-1)$-th order, $P_{5}$ a point with $\left(C_{2} . C_{3}\right)_{P_{5}}=2$, and $P_{6}$ an infinitely near point of $P_{5}$ on $C_{3}$ of the first order. Then,

$$
\begin{gathered}
I_{\tilde{X}}=\left\{\tilde{E}_{4}, \tilde{E}_{6}, \tilde{L}_{5,6}\right\}, \\
R_{\tilde{X}}=\left\{\tilde{L}=\tilde{L}_{1,2,5}, \tilde{E}_{1}, \tilde{E}_{2}, \tilde{E}_{3}, \tilde{E}_{5}\right\},
\end{gathered}
$$

so $X$ has the only $D_{5}$ singularity and contains exactly 3 lines.

2.4.3. Type $\langle 6\rangle$. Let $P_{1}$ be a point with $\left(C_{2} \cdot C_{3}\right)_{P_{1}}=6$, and $P_{i}(i=2, \ldots, 6)$ an infinitely near point of $P_{1}$ on $C_{3}$ of the $(i-1)$-th order. Then,

$$
I_{\tilde{X}}=\left\{\tilde{E}_{6}\right\}, R_{\tilde{X}}=\left\{\tilde{L}=\tilde{L}_{1,2,3}, \tilde{E}_{1}, \tilde{E}_{2}, \tilde{E}_{3}, \tilde{E}_{4}, \tilde{E}_{5}\right\}
$$

so $X$ has the only $E_{6}$ singularity and contains exactly one line.

\section{Normal FORMS AND THE NUMBER OF PARAMETERS}

In this section, we determine normal forms of all rational normal singular cubic surfaces and the number of parameters according to the type of singularity, slightly modifying the idea of [2].

3.1. Preliminaries. First we prepare some lemmata for the commutativity of blowing-up and blowing-down.

Lemma 3.1. Let $\sigma: S \rightarrow S^{\prime}$ be an isomorphism between smooth surfases with $\sigma(P)=Q$, where $P \in S$ and $Q \in S^{\prime}$ are points. Let $\varepsilon_{P}: S_{P} \rightarrow S$ (resp. $\varepsilon_{Q}: S_{Q}^{\prime} \rightarrow$ $\left.S^{\prime}\right)$ be the blowing-up at $P($ resp. $Q)$, and $E_{P}$ (resp. $\left.E_{Q}\right)$ the exceptional curve. Then there exists a unique isomorphism $\tilde{\sigma}: S_{P} \rightarrow S_{Q}^{\prime}$ with $\tilde{\sigma}\left(E_{P}\right)=E_{Q}$.

Proof. It follows from the universal property of blowing-up (see e.g. [1], [8]).

Lemma 3.2. Let $f: X \rightarrow Y$ and $f^{\prime}: X \rightarrow Y^{\prime}$ be surjective morphisms between normal projective varieties, and their fibres are connected. For any curve $C$ on $X$, we suppose that

$$
f(C) \text { is a point } \Longleftrightarrow f^{\prime}(C) \text { is a point . }
$$

Then there exists a unique isomorphism $g: Y \rightarrow Y^{\prime}$ such that $f^{\prime}=g \circ f$.

Proof. Let $X_{0}, Y_{0}, Y_{0}^{\prime}$ be the topological subspaces of $X, Y, Y^{\prime}$ respectively, consisting of all closed points, and let $f_{0}: X_{0} \rightarrow Y_{0}, f_{0}^{\prime}: X_{0} \rightarrow Y_{0}^{\prime}$ be induced maps. For a closed point $P \in Y$, the fibre $f^{-1}(P)$ is a projective scheme which is connected. Therefore, for any two closed points on the fibre, there exists a 1-dimensional closed subscheme which contains these two points. So we have a unique bijection $g_{0}: Y_{0} \rightarrow Y_{0}^{\prime}$ with $f_{0}^{\prime}=g_{0} \circ f_{0}$. Since a projective morphism is a closed morphism, a subset $U_{0}$ of $Y_{0}$ is an open set if and only if $f_{0}^{-1}\left(U_{0}\right)$ is an open set of $X_{0}$. Hence, $g_{0}$ is a homeomorphism. Since we can identify $Y, Y^{\prime}$ with the set of all 
irreducible closed subset, on $Y_{0}, Y_{0}^{\prime}$, respectively, we have a unique induced homeomorphism $g: Y \rightarrow Y^{\prime}$. Since $\mathcal{O}_{Y}=f_{*} \mathcal{O}_{X}, \mathcal{O}_{Y^{\prime}}=f_{*}^{\prime} \mathcal{O}_{X}, g$ is an isomorphism. (Cf. Kawamata [12.)

Lemma 3.3. Let $\tilde{\sigma}: S \rightarrow S^{\prime}$ be an isomorphism between smooth surfaces with $\tilde{\sigma}(E)=E^{\prime}$, where $E\left(\right.$ resp. $\left.E^{\prime}\right)$ is a $(-1)$-curve on $S$ (resp. $\left.S^{\prime}\right)$. Let $\varepsilon: S \rightarrow T$ be the blowing-down of $E$ to a point $P$, and $\varepsilon^{\prime}: S^{\prime} \rightarrow T^{\prime}$ the blowing-down of $E^{\prime}$ to a point $Q$. Then there exists a unique isomorphism $\sigma: T \rightarrow T^{\prime}$ with $\sigma(P)=Q$.

Proof. It follows from Lemma 3.2

3.2. The case of rank $f_{2}=3$. We determine the normal forms in the case of $\operatorname{rank} f_{2}=3$ with respect to each intersection symbol. First we recall a well-known fact for automorphisms on the projective plane.

Lemma 3.4. Let $C$ and $C^{\prime}$ be smooth conics in $\mathbf{P}^{2}$, and let $Q_{1}, Q_{2}, Q_{3}$ (resp. $\left.Q_{1}^{\prime}, Q_{2}^{\prime}, Q_{3}^{\prime}\right)$ be mutually different points on $C$ (resp. $\left.C^{\prime}\right)$. Then there exists a unique automorphism $\sigma$ on $\mathbf{P}^{2}$ with $\sigma(C)=C^{\prime}, \sigma\left(Q_{i}\right)=Q_{i}^{\prime}(i=1,2,3)$.

Proof. It follows from an easy calculation.

Hereafter, we put $f_{2}^{\prime}=x_{0} x_{2}-x_{1}^{2}$ and $C_{2}^{\prime}=\left\{f_{2}^{\prime}=0\right\} \subset \mathbf{P}^{2}$.

3.2.1. Type $1^{6}\left(A_{1}\right)$. For the fundamental points $P_{i}(i=1, \ldots, 6)$ of the birational map $\Phi: X-\rightarrow \mathbf{P}^{2}$ which are the points $C_{2} \cap C_{3}$, there exists a unique automorphism $\sigma_{0}$ on $\mathbf{P}^{2}$ with $\sigma_{0}\left(C_{2}\right)=C_{2}^{\prime}, \sigma_{0}\left(P_{1}\right)=(0: 0: 1), \sigma_{0}\left(P_{2}\right)=(1: 0: 0)$ and $\sigma_{0}\left(P_{3}\right)=(1: 1: 1)$, from Lemma 3.4. Then, we have $\sigma_{0}\left(P_{4}\right)=\left(a^{2}: a: 1\right)$, $\sigma_{0}\left(P_{5}\right)=\left(b^{2}: b: 1\right)$ and $\sigma_{0}\left(P_{6}\right)=\left(c^{2}: c: 1\right)$, where $a, b, c$ are three distinct elements of $\mathbf{C} \backslash\{0,1\}$. Let $X^{\prime}=\left\{F^{\prime}=x_{3} f_{2}^{\prime}-f_{3}^{\prime}=0\right\} \subset \mathbf{P}^{3}$, where

$$
f_{3}^{\prime}=\left(x_{0}-a x_{1}\right)\left(-x_{0}+(b+1) x_{1}-b x_{2}\right)\left(x_{1}-c x_{2}\right),
$$

and let $\Phi^{\prime}$ be a birational map defined by

$$
\Phi^{\prime}: \mathbf{P}^{2} \ni\left(y_{0}: y_{1}: y_{2}\right) \mapsto\left(y_{0} f_{2}^{\prime}\left(y_{i}\right): y_{1} f_{2}^{\prime}\left(y_{i}\right): y_{2} f_{2}^{\prime}\left(y_{i}\right): f_{3}^{\prime}\left(y_{i}\right)\right) \in X^{\prime}
$$

(see section 1). For $i=1, \ldots, 6, P_{i}^{\prime}:=\sigma_{0}\left(P_{i}\right)$ are fundamental points of $\Phi^{\prime}$. Let $S_{0}^{\prime}:=\mathbf{P}^{2}$, and let $\varepsilon_{i}: S_{i}^{\prime} \rightarrow S_{i-1}^{\prime}$ be the blowing-up at $P_{i}^{\prime}(i=1, \ldots, 6) . \sigma_{0}$ induces isomorphisms $\sigma_{i}: S_{i} \rightarrow S_{i}^{\prime}(i=1, \ldots, 6)$ by Lemma 3.1. As in section 11 there exists a birational morphism $\mu^{\prime}: S_{6}^{\prime} \rightarrow X^{\prime}$ which gives the minimal resolution of singularities, so we have an isomorphism $\tau: X \rightarrow X^{\prime}$ induced by $\sigma_{6}$, from Lemma 3.3. On the other hand, since $1^{6}$ is the only case where a singular cubic surface has the only $A_{1}$ singularity, the normal form of type $A_{1}$ is the polynomial $F^{\prime}$ above.

Hereafter, we put $X^{\prime}=\left\{x_{3} f_{2}^{\prime}-f_{3}^{\prime}=0\right\} \subset \mathbf{P}^{3}$ and determine the polynomial $f_{3}^{\prime}$ for any other case. We maintain the notation and conditions (for $P_{i}$ 's etc.) in section 2.2. according to intersection symbol.

3.2.2. Type $21^{4}\left(2 A_{1}\right)$. There exists a unique automorphism $\sigma_{0}$ with $\sigma_{0}\left(C_{2}\right)=C_{2}^{\prime}$, $\sigma_{0}\left(P_{1}\right)=(1: 1: 1)=: P_{1}^{\prime}, \sigma_{0}\left(P_{3}\right)=(0: 0: 1)=: P_{3}^{\prime}$ and $\sigma_{0}\left(P_{4}\right)=(1: 0: 0)=: P_{4}^{\prime}$. Then, we have $\sigma_{0}\left(P_{5}\right)=\left(a^{2}: a: 1\right)=: P_{5}^{\prime}$ and $\sigma_{0}\left(P_{6}\right)=\left(b^{2}: b: 1\right)=: P_{6}^{\prime}$, where $a, b$ are two distinct elements of $\mathbf{C} \backslash\{0,1\}$. Let

$$
f_{3}^{\prime}=\left(x_{0}-2 x_{1}+x_{2}\right)\left(x_{0}-a x_{1}\right)\left(x_{1}-b x_{2}\right),
$$

and let $P_{2}^{\prime}$ be an infinitely near point of $P_{1}^{\prime}$ on $C_{2}^{\prime}$ of the first order. We define $\Phi^{\prime}, S_{i}^{\prime}$ as above. Then, $P_{i}^{\prime}(i=1, \ldots, 6)$ are fundamental points of $\Phi^{\prime}$. Since $\sigma_{1}\left(P_{2}\right)=P_{2}^{\prime}$, 
$\sigma_{0}$ induces isomorphisms $\sigma_{i}: S_{i} \rightarrow S_{i}^{\prime}(i=1, \ldots, 6)$ by Lemma 3.1] In the same way as above, we have an isomorphism $\tau: X \rightarrow X^{\prime}$ induced by $\sigma_{6}$.

3.2.3. Type $31^{3}\left(A_{1} A_{2}\right)$. There exists a unique automorphism $\sigma_{0}$ on $\mathbf{P}^{2}$ with $\sigma_{0}\left(C_{2}\right)$ $=C_{2}^{\prime}, \sigma_{0}\left(P_{1}\right)=(1: 1: 1), \sigma_{0}\left(P_{4}\right)=(0: 0: 1)$ and $\sigma_{0}\left(P_{5}\right)=(1: 0: 0)$. Then, we have $\sigma_{0}\left(P_{6}\right)=\left(a^{2}: a: 1\right)$, where $a$ is an element of $\mathbf{C} \backslash\{0,1\}$. Putting

$$
f_{3}^{\prime}=\left(x_{0}-x_{1}\right)\left(-x_{1}+x_{2}\right)\left(x_{0}-(a+1) x_{1}+a x_{2}\right),
$$

we similarly have an isomorphism between $X$ and $X^{\prime}$.

3.2.4. Type $2^{2} 1^{2}\left(3 A_{1}\right)$. There exists a unique automorphism $\sigma_{0}$ on $\mathbf{P}^{2}$ with $\sigma_{0}\left(C_{2}\right)$ $=C_{2}^{\prime}, \sigma_{0}\left(P_{1}\right)=(0: 0: 1), \sigma_{0}\left(P_{3}\right)=(1: 0: 0)$ and $\sigma_{0}\left(P_{5}\right)=(1: 1: 1)$. Then, $\sigma_{0}\left(P_{6}\right)=\left(a^{2}: a: 1\right)$, where $a$ is an element of $\mathbf{C} \backslash\{0,1\}$. We have an isomorphism between $X$ and $X^{\prime}$ by putting

$$
f_{3}^{\prime}=x_{0} x_{2}\left(x_{0}-(a+1) x_{1}+a x_{2}\right) .
$$

3.2.5. Type $41^{2}\left(A_{1} A_{3}\right)$. There exists a unique automorphism $\sigma_{0}$ on $\mathbf{P}^{2}$ with $\sigma_{0}\left(C_{2}\right)$ $=C_{2}^{\prime}, \sigma_{0}\left(P_{1}\right)=(1: 1: 1), \sigma_{0}\left(P_{5}\right)=(0: 0: 1)$ and $\sigma\left(P_{6}\right)=(1: 0: 0)$. We have an isomorphism between $X$ and $X^{\prime}$ by putting

$$
f_{3}^{\prime}=\left(x_{0}-x_{1}\right)\left(-x_{1}+x_{2}\right)\left(x_{0}-2 x_{1}+x_{2}\right) .
$$

3.2.6. Type $321\left(2 A_{1} A_{2}\right)$. There exists a unique automorphism $\sigma_{0}$ on $\mathbf{P}^{2}$ with $\sigma_{0}\left(C_{2}\right)=C_{2}^{\prime}, \sigma_{0}\left(P_{1}\right)=(0: 0: 1), \sigma_{0}\left(P_{4}\right)=(1: 0: 0)$ and $\sigma_{0}\left(P_{6}\right)=(1: 1: 1)$. We have an isomorphism between $X$ and $X^{\prime}$ by putting

$$
f_{3}^{\prime}=x_{1}^{2}\left(x_{0}-x_{1}\right) .
$$

3.2.7. Type $2^{3}\left(4 A_{1}\right)$. There exists a unique automorphism $\sigma_{0}$ on $\mathbf{P}^{2}$ with $\sigma_{0}\left(C_{2}\right)=$ $C_{2}^{\prime}, \sigma_{0}\left(P_{1}\right)=(0: 0: 1), \sigma_{0}\left(P_{3}\right)=(1: 0: 0)$ and $\sigma_{0}\left(P_{5}\right)=(1: 1: 1)$. We have an isomorphism between $X$ and $X^{\prime}$ by putting

$$
f_{3}^{\prime}=\left(x_{0}-x_{1}\right)\left(x_{1}-x_{2}\right) x_{1} .
$$

3.2.8. Type $51\left(A_{1} A_{4}\right)$. There exists an automorphism $\sigma_{0}$ on $\mathbf{P}^{2}$ with $\sigma_{0}\left(C_{2}\right)=C_{2}^{\prime}$, $\sigma_{0}\left(P_{1}\right)=(0: 0: 1)$ and $\sigma_{0}\left(P_{6}\right)=(1: 0: 0)$. We have an isomorphism between $X$ and $X^{\prime}$ by putting

$$
f_{3}^{\prime}=x_{0}^{2} x_{1} .
$$

3.2.9. Type $42\left(2 A_{1} A_{3}\right)$. There exists an automorphism $\sigma_{0}$ on $\mathbf{P}^{2}$ with $\sigma_{0}\left(C_{2}\right)=C_{2}^{\prime}$, $\sigma_{0}\left(P_{1}\right)=(0: 0: 1)$ and $\sigma_{0}\left(P_{5}\right)=(1: 0: 0)$. We have an isomorphism between $X$ and $X^{\prime}$ by putting

$$
f_{3}^{\prime}=x_{0} x_{1}^{2} .
$$

3.2.10. Type $3^{2}\left(A_{1} 2 A_{2}\right)$. There exists an automorphism $\sigma_{0}$ on $\mathbf{P}^{2}$ with $\sigma_{0}\left(C_{2}\right)=$ $C_{2}^{\prime}, \sigma_{0}\left(P_{1}\right)=(0: 0: 1)$ and $\sigma_{0}\left(P_{4}\right)=(1: 0: 0)$. We have an isomorphism between $X$ and $X^{\prime}$ by putting

$$
f_{3}^{\prime}=x_{1}^{3} .
$$

3.2.11. Type $6\left(A_{1} A_{5}\right)$. There exists an automorphism $\sigma_{0}$ on $\mathbf{P}^{2}$ with $\sigma_{0}\left(C_{2}\right)=C_{2}^{\prime}$ and $\sigma_{0}\left(P_{1}\right)=(0: 0: 1)$. We have an isomorphism between $X$ and $X^{\prime}$ by putting

$$
f_{3}^{\prime}=x_{0}^{3} \text {. }
$$


3.3. The case of rank $f_{2}=2$. We determine the normal forms in the case of $\operatorname{rank} f_{2}=2$ with respect to each intersection symbol. First, we recall well-known facts for automorphisms on the projective plane.

Lemma 3.5. Let $Q_{1}, \ldots, Q_{4}$ (resp. $\left.Q_{1}^{\prime}, \ldots, Q_{4}^{\prime}\right)$ be points on $\mathbf{P}^{2}$. Assume that no 3 of the $Q_{i}$ (resp. $\left.Q_{i}^{\prime}\right)$ are collinear. Then there exists a unique automorphism $\sigma$ on $\mathbf{P}^{2}$ with $\sigma\left(Q_{i}\right)=Q_{i}^{\prime}(i=1,2,3,4)$.

Proof. It follows from an easy calculation.

Lemma 3.6. Let $Q_{1}, Q_{2}$ and $Q_{3}$ be points on $\mathbf{P}^{2}$. Assume that they are on a line $L$. If an automorphism $\sigma$ on $\mathbf{P}^{2}$ fixes $Q_{1}, Q_{2}$ and $Q_{3}$, then $\left.\sigma\right|_{L}=\mathrm{id}_{L}$.

Proof. It follows from an easy calculation.

We put $f_{2}^{\prime}=x_{0} x_{1}$ and $C_{2}^{\prime}=\left\{f_{2}^{\prime}=0\right\}$, and maintain the notation and conditions (for $P_{i}$ 's, etc.) in section 2.3 according to the intersection symbol.

3.3.1. Type $1^{3} \cdot 1^{3}\left(A_{2}\right)$. There exists a unique automorphism $\sigma_{0}$ on $\mathbf{P}^{2}$ with $\sigma_{0}\left(P_{1}\right)$ $=(0: 1: 0), \sigma_{0}\left(P_{2}\right)=(0: 1:-1), \sigma_{0}\left(P_{4}\right)=(1: 0: 0)$ and $\sigma_{0}\left(P_{5}\right)=(1: 0:-1)$. Then, we have $\sigma_{0}\left(P_{3}\right)=(0: d: 1)$ and $\sigma_{0}\left(P_{6}\right)=(e: 0: 1)$, where $d, e$ are elements of $\mathbf{C} \backslash\{0,-1\}$. In the same way as above, $X$ is isomorphic to $X^{\prime}=\left\{x_{3} f_{2}^{\prime}-f_{3}^{\prime}=0\right\}$ by putting

$$
f_{3}^{\prime}=x_{2}\left(x_{0}+x_{1}+x_{2}\right)\left(d x_{0}+e x_{1}-d e x_{2}\right) .
$$

3.3.2. Type $1^{3} .21\left(A_{2} A_{1}\right)$. There exists a unique automorphism $\sigma_{0}$ on $\mathbf{P}^{2}$ with $\sigma_{0}\left(P_{1}\right)=(0: 1: 0), \sigma_{0}\left(P_{2}\right)=(0: 1:-1), \sigma_{0}\left(P_{4}\right)=(1: 0: 0)$ and $\sigma_{0}\left(P_{6}\right)=(1:$ $0:-1)$. Then, we have $\sigma_{0}\left(P_{3}\right)=(0: d: 1)$, where $d$ is an element of $\mathbf{C} \backslash\{0,-1\}$. Similarly, we have an isomorphism between $X$ and $X^{\prime}$ by putting

$$
f_{3}^{\prime}=x_{2}\left(x_{1}-d x_{2}\right)\left(x_{0}+x_{1}+x_{2}\right) .
$$

However, $X^{\prime}$ is isomorphic to

$$
X^{\prime \prime}=\left\{x_{3}\left(x_{0} x_{2}-x_{1}^{2}\right)-\left(x_{0}-x_{1}\right)\left(-x_{1}+x_{2}\right)\left(x_{0}-(a+1) x_{1}+a x_{2}\right)=0\right\}
$$

(cf. subsection 3.2.3) by the projective transformation

$$
X^{\prime \prime} \ni\left(x_{0}: x_{1}: x_{2}: x_{3}\right) \mapsto\left(d x_{3}:-d x_{0}+2 d x_{1}-d x_{2}: x_{1}-x_{2}: x_{1}\right) \in X^{\prime},
$$

where $a=1+1 / d$. Therefore, the normal form of a singular cubic surface which has $A_{1} A_{2}$ singularities is given as in subsection 3.2 .3

3.3.3. Type $1^{3} .3\left(2 A_{2}\right)$. We must take care in this case. There exists an automorphism $\sigma_{0}$ on $\mathbf{P}^{2}$ with $\sigma_{0}\left(P_{1}\right)=(0: 1: 0)=: P_{1}^{\prime}, \sigma_{0}\left(P_{2}\right)=(0: 1:-1)=: P_{2}^{\prime}$, $\sigma_{0}\left(P_{4}\right)=(1: 0: 0)=: P_{4}^{\prime}$ and $\sigma_{4}\left(P_{5}\right)=P_{5}^{\prime}$, where $P_{5}^{\prime}$ is an infinitely near point of $P_{4}^{\prime}$ on $C_{2}^{\prime}$ of the first order. Let $P_{6}^{\prime}$ be an infinitely near point of $P_{4}^{\prime}$ on $C_{2}^{\prime}$ of the second order. Of course, the line which passes through $P_{4}^{\prime}$ and $P_{5}^{\prime}$ (i.e., the line $x_{1}=0$ ) also passes through $P_{6}^{\prime}$. We denote this line by $L_{4,5,6}^{\prime}$. Since $\sigma_{0}$ satisfies $\sigma_{0}\left(L_{4,5,6}\right)=L_{4,5,6}^{\prime}$, any automorphism satisfying the conditions above sends $O=L_{1,2,3} \cap L_{4,5,6}$ to $O^{\prime}=L_{1,2,3}^{\prime} \cap L_{4,5,6}^{\prime}$, where $L_{1,2,3}^{\prime}$ is a line that passes through $P_{1}^{\prime}, P_{2}^{\prime}$ and $P_{3}^{\prime}$ (i.e., the line $\left.x_{0}=0\right)$. Therefore, $\sigma_{0}$ sends $P_{3}$ to $P_{3}^{\prime}:=(0: d: 1)$, where $d$ is an element of $\mathbf{C} \backslash\{0,-1\}$, and $d$ does not depend on a choice of $\sigma_{0}$, by Lemma 3.6. In the same way as above, we have an isomorphism between $X$ and $X^{\prime}$ by putting

$$
f_{3}^{\prime}=x_{2}\left(x_{1}+x_{2}\right)\left(-x_{1}+d x_{2}\right) .
$$


3.3.4. Type $21.21\left(A_{2} 2 A_{1}\right)$. There exists a unique automorphism $\sigma_{0}$ on $\mathbf{P}^{2}$ with $\sigma_{0}\left(P_{1}\right)=(0: 1: 0), \sigma_{0}\left(P_{3}\right)=(0: 1:-1), \sigma_{0}\left(P_{4}\right)=(1: 0: 0)$ and $\sigma_{0}\left(P_{6}\right)=(1: 0:$ $-1)$. We have an isomorphism between $X$ and $X^{\prime}$ by putting

$$
f_{3}^{\prime}=x_{2}^{2}\left(x_{0}+x_{1}+x_{2}\right) \text {. }
$$

However, $X^{\prime}$ is isomorphic to

$$
X^{\prime \prime}=\left\{x_{3}\left(x_{0} x_{2}-x_{1}^{2}\right)-x_{1}^{2}\left(x_{0}-x_{1}\right)=0\right\}
$$

(cf. subsection 3.2.6) by the projective transformation

$$
X^{\prime \prime} \ni\left(x_{0}: x_{1}: x_{2}: x_{3}\right) \mapsto\left(x_{3}: x_{0}:-x_{1}: x_{2}\right) \in X^{\prime} .
$$

3.3.5. Type $21.3\left(2 A_{2} A_{1}\right)$. There exists an automorphism $\sigma_{0}$ on $\mathbf{P}^{2}$ with $\sigma_{0}\left(P_{1}\right)=$ $(0: 1: 0), \sigma_{0}\left(P_{3}\right)=(0: 1:-1), \sigma_{0}\left(P_{4}\right)=(1: 0: 0)=: P_{4}^{\prime}$ and $\sigma_{4}\left(P_{5}\right)=P_{5}^{\prime}$, where $P_{5}^{\prime}$ is an infinitely near point of $P_{4}^{\prime}$ on $C_{2}^{\prime}$ of the first order. We have an isomorphism between $X$ and $X^{\prime}$ by putting

$$
f_{3}^{\prime}=x_{2}^{2}\left(x_{1}+x_{2}\right) .
$$

However, $X^{\prime}$ is isomorphic to

$$
X^{\prime \prime}=\left\{x_{3}\left(x_{0} x_{2}-x_{1}^{2}\right)-x_{1}^{3}=0\right\}
$$

(cf. subsection 3.2.10) by the projective transformation

$$
X^{\prime \prime} \ni\left(x_{0}: x_{1}: x_{2}: x_{3}\right) \mapsto\left(x_{0}: x_{3}: x_{1}: x_{2}\right) \in X^{\prime} .
$$

3.3.6. Type $3.3\left(3 A_{2}\right)$. There exists an automorphism $\sigma_{0}$ on $\mathbf{P}^{2}$ with $\sigma_{0}\left(P_{1}\right)=(0$ : $1: 0)=: P_{1}^{\prime}, \sigma_{0}\left(P_{4}\right)=(1: 0: 0)=: P_{4}^{\prime}, \sigma_{1}\left(P_{2}\right)=P_{2}^{\prime}$ and $\sigma_{4}\left(P_{5}\right)=P_{5}^{\prime}$, where $P_{2}^{\prime}$ (resp. $P_{5}^{\prime}$ ) is an infinitely near point of $P_{1}^{\prime}$ (resp. $P_{4}^{\prime}$ ) on $C_{2}^{\prime}$ of the first order. We have an isomorphism between $X$ and $X^{\prime}$ by putting

$$
f_{3}^{\prime}=x_{2}^{3} \text {. }
$$

3.3.7. Type $1^{2} .1^{2}\left(A_{3}\right)$. There exists a unique automorphism $\sigma_{0}$ on $\mathbf{P}^{2}$ with $\sigma_{0}\left(P_{1}\right)$ $=(0: 1: 0), \sigma_{0}\left(P_{2}\right)=(0: 1:-1), \sigma_{0}\left(P_{3}\right)=(1: 0: 0)$ and $\sigma_{0}\left(P_{4}\right)=(1: 0:-1)$. Then, we have $\sigma_{0}\left(P_{5}\right)=(0: 0: 1)=: P_{5}^{\prime}$ and $\sigma_{5}\left(P_{6}\right)=P_{6}^{\prime}$, where $P_{6}^{\prime}$ is an infinitely near point of $P_{5}^{\prime}$ on $x_{0}-u x_{1}=0$ of the first order, and $u$ is an element of $\mathbf{C}^{\times}$. We have an isomorphism between $X$ and $X^{\prime}$ by putting

$$
f_{3}^{\prime}=x_{2}\left(x_{0}+x_{1}+x_{2}\right)\left(x_{0}-u x_{1}\right) .
$$

3.3.8. Type $1^{2} .2\left(A_{3} A_{1}\right)$. There exists a unique automorphism $\sigma_{0}$ on $\mathbf{P}^{2}$ with $\sigma_{0}\left(P_{1}\right)$ $=(0: 1: 1), \sigma_{0}\left(P_{2}\right)=(0: 1:-1), \sigma_{0}\left(P_{3}\right)=(1: 0: 0)=: P_{3}^{\prime}, \sigma_{3}\left(P_{4}\right)=P_{4}^{\prime}$ and $\sigma_{5}\left(P_{6}\right)=P_{6}^{\prime}$, where $P_{4}^{\prime}$ is an infinitely near point of $P_{3}^{\prime}$ on $C_{2}^{\prime}$ of the first order, and $P_{6}^{\prime}$ is an infinitely near point of $P_{5}^{\prime}:=(0: 0: 1)$ on

$$
f_{3}^{\prime}=x_{0} x_{2}^{2}-x_{1}^{3}+x_{1} x_{2}^{2}=0
$$

of the first order. Similarly, we have an isomorphism between $X$ and $X^{\prime}$.

However, $X^{\prime}$ is isomorphic to

$$
X^{\prime \prime}=\left\{x_{3}\left(x_{0} x_{2}-x_{1}^{2}\right)-\left(x_{0}-x_{1}\right)\left(-x_{1}+x_{2}\right)\left(x_{0}-2 x_{1}+x_{2}\right)=0\right\}
$$

(cf. subsection 3.2.5) by the projective transformation

$$
X^{\prime \prime} \ni\left(x_{0}: x_{1}: x_{2}: x_{3}\right) \mapsto\left(-x_{3}: x_{0}-2 x_{1}+x_{2}: x_{0}-x_{2}: x_{0}+2 x_{1}+x_{2}\right) \in X^{\prime} .
$$


3.3.9. Type $2.2\left(A_{3} 2 A_{1}\right)$. There exists an automorphism $\sigma_{0}$ on $\mathbf{P}^{2}$ with $\sigma_{0}\left(P_{1}\right)=$ $(0: 1: 0)=: P_{1}^{\prime}, \sigma_{1}\left(P_{2}\right)=P_{2}^{\prime}, \sigma_{0}\left(P_{3}\right)=(1: 0: 0)=: P_{3}^{\prime}, \sigma_{3}\left(P_{4}\right)=P_{4}^{\prime}, \sigma_{0}\left(P_{5}\right)=$ $(0: 0: 1)=: P_{5}^{\prime}$ and $\sigma_{5}\left(P_{6}\right)=P_{6}^{\prime}$, where $P_{2}^{\prime}$ (resp. $\left.P_{4}^{\prime}\right)$ is an infinitely near point of $P_{1}^{\prime}$ (resp. $P_{3}^{\prime}$ ) on $C_{2}^{\prime}$ of the first order, and $P_{6}^{\prime}$ is an infinitely near point of $P_{5}^{\prime}$ on

$$
f_{3}^{\prime}=x_{2}^{2}\left(x_{0}+x_{1}\right)=0
$$

of the first order. Similarly, we have an isomorphism between $X$ and $X^{\prime}$.

However, $X^{\prime}$ is isomorphic to

$$
X^{\prime \prime}=\left\{x_{3}\left(x_{0} x_{2}-x_{1}^{2}\right)-x_{0} x_{1}^{2}=0\right\}
$$

(cf. subsection 3.2.9) by the projective transformation

$$
X^{\prime \prime} \ni\left(x_{0}: x_{1}: x_{2}: x_{3}\right) \mapsto\left(x_{0}: x_{3}: x_{1}: x_{2}\right) \in X^{\prime} .
$$

3.3.10. Type $1^{2} .1\left(A_{4}\right)$. There exists a unique automorphism $\sigma_{0}$ on $\mathbf{P}^{2}$ with $\sigma_{0}\left(P_{1}\right)$ $=(0: 1: 1), \sigma_{0}\left(P_{2}\right)=(0: 1:-1), \sigma_{0}\left(P_{3}\right)=(1: 0: 0), \sigma_{0}\left(P_{4}\right)=(0: 0: 1)=$ : $P_{4}^{\prime}, \sigma_{4}\left(P_{5}\right)=P_{5}^{\prime}$ and $\sigma_{5}\left(P_{6}\right)=P_{6}^{\prime}$, where $P_{5}^{\prime}$ is an infinitely near point of $P_{4}^{\prime}$ on

$$
f_{3}^{\prime}=x_{0}^{2} x_{2}+x_{1}^{3}-x_{1} x_{2}^{2}=0
$$

of the first order, and $P_{6}^{\prime}$ is an infinitely near point of $P_{4}^{\prime}$ on $f_{3}^{\prime}=0$ of the second order. Similarly, we have an isomorphism between $X$ and $X^{\prime}$.

3.3.11. Type $2.1\left(A_{4} A_{1}\right)$. Let $P_{1}^{\prime}:=(0: 1: 0), P_{3}^{\prime}:=(1: 0: 0), P_{4}^{\prime}:=(0: 0: 1), P_{2}^{\prime}$ be an infinitely near point of $P_{1}^{\prime}$ on $C_{2}^{\prime}$ of the first order, and let $P_{i}^{\prime}(i=5,6)$ be an infinitely near point of $P_{4}^{\prime}$ on

$$
f_{3}^{\prime}=x_{2}\left(x_{0}^{2}+x_{1} x_{2}\right)=0
$$

of the $(i-4)$-th order. There exists an automorphism $\sigma_{0}$ on $\mathbf{P}^{2}$ with $\sigma_{0}\left(P_{1}\right)=P_{1}^{\prime}$, $\sigma_{0}\left(P_{3}\right)=P_{3}^{\prime}, \sigma_{0}\left(P_{4}\right)=P_{4}^{\prime}$, and $\sigma_{5}\left(P_{6}\right)=P_{6}^{\prime}$. Then, we have $\sigma_{1}\left(P_{2}\right)=P_{2}^{\prime}$ and $\sigma_{4}\left(P_{5}\right)=P_{5}^{\prime}$. Similarly, we have an isomorphism between $X$ and $X^{\prime}$.

However, $X^{\prime}$ is isomorphic to

$$
X^{\prime \prime}=\left\{x_{3}\left(x_{0} x_{2}-x_{1}^{2}\right)-x_{0}^{2} x_{1}=0\right\}
$$

(cf. subsection 3.2.8) by the projective transformation

$$
X^{\prime \prime} \ni\left(x_{0}: x_{1}: x_{2}: x_{3}\right) \mapsto\left(x_{0}: x_{3}: x_{1}: x_{2}\right) \in X^{\prime} .
$$

3.3.12. Type $1^{2} .0\left(A_{5}\right)$. Let $P_{1}^{\prime}:=(0: 1: 1), P_{2}^{\prime}:=(0: 1:-1), P_{3}^{\prime}:=(0: 0: 1)$, and let $P_{i}^{\prime}(i=4,5,6)$ be an infinitely near point of $P_{3}^{\prime}$ on

$$
f_{3}^{\prime}=x_{0}^{3}+x_{1}^{3}-x_{1} x_{2}^{2}=0
$$

of the $(i-3)$-th order. There exists an automorphism $\sigma_{0}$ on $\mathbf{P}^{2}$ with $\sigma_{0}\left(P_{1}\right)=P_{1}^{\prime}$, $\sigma_{0}\left(P_{2}\right)=P_{2}^{\prime}, \sigma_{0}\left(P_{3}\right)=P_{3}^{\prime}, \sigma_{3}\left(P_{4}\right)=P_{4}^{\prime}, \sigma_{5}\left(P_{6}\right)=P_{6}^{\prime}$ and $\sigma_{4}\left(P_{5}\right)=P_{5}^{\prime}$. Similarly, we have an isomorphism between $X$ and $X^{\prime}$. 
3.3.13. Type $2.0\left(A_{5} A_{1}\right)$. Let $P_{1}^{\prime}:=(0: 1: 0), P_{2}^{\prime}$ be an infinitely near point of $P_{1}^{\prime}$ on $C_{2}^{\prime}$ of the first order, $P_{3}^{\prime}:=(0: 0: 1)$, and let $P_{i}^{\prime}(i=4,5,6)$ be an infinitely near point of $P_{3}^{\prime}$ on

$$
f_{3}^{\prime}=x_{0}^{3}+x_{1} x_{2}^{2}=0
$$

of the $(i-3)$-th order. There exists an automorphism $\sigma_{0}$ on $\mathbf{P}^{2}$ with $\sigma_{0}\left(P_{1}\right)=P_{1}^{\prime}$, $\sigma_{0}\left(P_{3}\right)=P_{3}^{\prime}, \sigma_{3}\left(P_{4}\right)=P_{4}^{\prime}, \sigma_{5}\left(P_{6}\right)=P_{6}^{\prime}, \sigma_{1}\left(P_{2}\right)=P_{2}^{\prime}$ and $\sigma_{4}\left(P_{5}\right)=P_{5}^{\prime}$. Similarly, we have an isomorphism between $X$ and $X^{\prime}$.

However, $X^{\prime}$ is isomorphic to

$$
X^{\prime \prime}=\left\{x_{3}\left(x_{0} x_{2}-x_{1}^{2}\right)-x_{0}^{3}=0\right\}
$$

(cf. subsection 3.2.11) by the projective transformation

$$
X^{\prime \prime} \ni\left(x_{0}: x_{1}: x_{2}: x_{3}\right) \mapsto\left(x_{0}: x_{3}: x_{1}: x_{2}\right) \in X^{\prime} .
$$

3.4. The case of rank $f_{2}=1$. We determine the normal forms in the case of rank $f_{2}=1$ (however these are already given in [2]). We maintain the notation and condition (for $P_{i}$ 's, etc.) in section 2.4, according to intersection symbol. We put $f_{2}^{\prime}=x_{0}^{2}$ and $C_{2}^{\prime}=\left\{f_{2}^{\prime}=0\right\}$.

3.4.1. Type $\left\langle 2^{3}\right\rangle\left(D_{4}\right)$. We must take care in this case, that is, there are two normal forms according to the position of $P_{i}$.

(1) In the case where $L_{1,2}, L_{3,4}$ and $L_{5,6}$ meet at a point $Q$, we can say that $P_{6}$ is an infinitely near point of $P_{5}$ on a line, which passes through $P_{5}$ and $Q$. Let $P_{1}^{\prime}:=(0: 1:-1), P_{3}^{\prime}:=(0: 1:-\omega)$, and $P_{5}^{\prime}:=\left(0: 1:-\omega^{2}\right)$, where $\omega^{3}=1(\omega \neq 1)$, and let $P_{i}^{\prime}(i=2,4,6)$ be an infinitely near point of $P_{i-1}^{\prime}$ on

$$
f_{3}^{\prime}=x_{1}^{3}+x_{2}^{3}=0
$$

of the first order. There exists an automorphism $\sigma_{0}$ satisfying $\sigma_{0}\left(P_{1}\right)=P_{1}^{\prime}$, $\sigma_{0}\left(P_{3}\right)=P_{3}^{\prime}, \sigma_{0}\left(P_{5}\right)=P_{5}^{\prime}, \sigma_{1}\left(P_{2}\right)=P_{2}^{\prime}$ and $\sigma_{3}\left(P_{4}\right)=P_{4}^{\prime}$. Then we have $\sigma_{0}(Q)=(1: 0: 0)$. Since $L_{5,6}^{\prime}$ passes through $(1: 0: 0)$, where $L_{5,6}^{\prime}$ is a line that passes through $P_{5}^{\prime}$ and $P_{6}^{\prime}$ (i.e., the line $x_{1}+\omega x_{2}=0$ ), we have $\sigma_{0}\left(L_{5,6}\right)=L_{5,6}^{\prime}$. Hence, $\sigma_{0}$ sends $P_{6}$ to $P_{6}^{\prime}$. In the same way as above, $X$ is isomorphic to $X^{\prime}$.

(2) In the case where $L_{1,2}, L_{3,4}$ and $L_{5,6}$ do not meet at a point, we put $P_{1}^{\prime}:=(0: 1:-1), P_{3}^{\prime}:=(0: 1:-\omega), P_{5}^{\prime}:=\left(0: 1:-\omega^{2}\right)$. Also let $P_{i}^{\prime}(i=2,4,6)$ be an infinitely near point of $P_{i-1}^{\prime}$ on

$$
f_{3}^{\prime}=x_{1}^{3}+x_{2}^{3}+x_{0} x_{1} x_{2}=0
$$

of the first order. There exists a unique automorphism $\sigma_{0}$ satisfying $\sigma_{0}\left(P_{1}\right)$ $=P_{1}^{\prime}, \sigma_{0}\left(P_{3}\right)=P_{3}^{\prime}, \sigma_{0}\left(P_{5}\right)=P_{5}^{\prime}, \sigma_{1}\left(P_{2}\right)=P_{2}^{\prime}, \sigma_{3}\left(P_{4}\right)=P_{4}^{\prime}$ and $\sigma_{5}\left(P_{6}\right)=$ $P_{6}^{\prime}$. Similarly, $X$ is isomorphic to $X^{\prime}$.

3.4.2. Type $\langle 42\rangle\left(D_{5}\right)$. We put

$$
f_{3}^{\prime}=x_{0} x_{2}^{2}+x_{1}^{2} x_{2}
$$

and $C_{3}^{\prime}=\left\{f_{3}^{\prime}=0\right\}$. Let $P_{1}^{\prime}:=(0: 0: 1)$ and let $P_{i}^{\prime}(i=2,3,4)$ be an infinitely near point of $P_{1}^{\prime}$ on $C_{3}^{\prime}$ of the $(i-1)$-th order, $P_{5}^{\prime}:=(0: 1: 0)$, and let $P_{6}^{\prime}$ be an infinitely near point of $P_{5}^{\prime}$ on $C_{3}^{\prime}$ of the first order. There exists an automorphism $\sigma_{0}$ on $\mathbf{P}^{2}$ with $\sigma_{0}\left(P_{1}\right)=P_{1}^{\prime}, \sigma_{0}\left(P_{5}\right)=P_{5}^{\prime}, \sigma_{2}\left(P_{3}\right)=P_{3}^{\prime}, \sigma_{3}\left(P_{4}\right)=P_{4}^{\prime}$ and $\sigma_{5}\left(P_{6}\right)=P_{6}^{\prime}$. Then, we have $\sigma_{1}\left(P_{2}\right)=P_{2}^{\prime}$. Similarly, $X$ is isomorphic to $X^{\prime}$. 
3.4.3. Type $\langle 6\rangle\left(E_{6}\right)$. We put

$$
f_{3}^{\prime}=x_{0} x_{2}^{2}+x_{1}^{3}
$$

and $C_{3}^{\prime}=\left\{f_{3}^{\prime}=0\right\}$. Let $P_{1}^{\prime}:=(0: 0: 1)$, and let $P_{i}^{\prime}(i=2, \ldots, 6)$ be an infinitely near point of $P_{1}^{\prime}$ on $C_{3}^{\prime}$ of the $(i-1)$-th order. There exists an automorphism $\sigma_{0}$ on $\mathbf{P}^{2}$ with $\sigma_{0}\left(P_{1}\right)=P_{1}^{\prime}, \sigma_{1}\left(P_{2}\right)=P_{2}^{\prime}, \sigma_{3}\left(P_{4}\right)=P_{4}^{\prime}, \sigma_{4}\left(P_{5}\right)=P_{5}^{\prime}$ and $\sigma_{5}\left(P_{6}\right)=P_{6}^{\prime}$. Then, we have $\sigma_{2}\left(P_{3}\right)=P_{3}^{\prime}$. Similarly, $X$ is isomorphic to $X^{\prime}$.

\section{Proof of Theorem 3}

In this section, we determine automorphism groups on normal singular cubic surfaces with no parameters.

\subsection{Preliminaries.}

Proposition 4.1. Let $X$ be a rational normal singular cubic surface and let $\mu$ : $\tilde{X} \rightarrow X$ be the minimal resolution of singularities. Then, there exists a group isomorphism $\gamma$ : Aut $X \simeq$ Aut $\tilde{X}$.

Proof. We define a group homomorphism $\gamma:$ Aut $X \rightarrow$ Aut $\tilde{X}$ as follows. For any $\sigma \in$ Aut $X$, we show that the birational map $\tilde{\sigma}:=\mu^{-1} \circ \sigma \circ \mu$ is an automorphism on $\tilde{X}$. If $\tilde{\sigma}$ has some fundamental points, then we consider the elimination of indeterminacy of $\tilde{\sigma}$. We note that $\sigma$ sends the singular points of $X$ to the singular points of $X$ and that the fundamental points of $\tilde{\sigma}$ are on (-2)-curves. We assume that $\tilde{\sigma}$ has $m$ fundamental points (possibly infinitely near). We can eliminate the indeterminacy of $\sigma$ by $m$ blowings-up. Let $g: \tilde{X}^{\prime} \rightarrow \tilde{X}$ be a composition of their blowings-up. Then we have a birational morphism $\varphi:=\tilde{\sigma} \circ g$. For the exceptional curve $E$ of the $m$-th blowing-up, $\varphi(E)$ is one of $(-2)$-curves. However, the self-intersection number does not decrease by a birational morphism, so it is a contradiction. Therefore, $\tilde{\sigma}$ is an automorphism on $\tilde{X}$. We define a map $\gamma$ : Aut $X \rightarrow$ Aut $\tilde{X}$ by this correspondence. Obviously, $\gamma$ is a group homomorphism.

It is clear that $\gamma$ is injective. We prove that $\gamma$ is surjective. For $\tilde{\sigma} \in$ Aut $\tilde{X}$, there exists a unique automorphism $\sigma \in \operatorname{Aut} X$ with $\mu \circ \tilde{\sigma}=\sigma \circ \mu$ by Lemma 3.2 and we have $\gamma(\sigma)=\tilde{\sigma}$. So $\gamma$ is surjective.

Definition 4.2. For a subset $\Gamma_{\tilde{X}}:=R_{\tilde{X}} \cup I_{\tilde{X}}$ of $\operatorname{Div} \tilde{X}$, we define the automorphism group of configuration of $\Gamma_{\tilde{X}}$, Aut $\Gamma_{\tilde{X}}$, to be the group of permutations of $\Gamma_{\tilde{X}}$ preserving the relation of intersection. There exists a group homomorphism $\Psi$ : Aut $\tilde{X} \rightarrow \operatorname{Aut} \Gamma_{\tilde{X}}$, which sends $\tilde{\sigma} \in$ Aut $\tilde{X}$ to a permutation of $\Gamma_{\tilde{X}}$ induced by the pullback $\left(\tilde{\sigma}^{-1}\right)^{*}: \operatorname{Div} \tilde{X} \rightarrow \operatorname{Div} \tilde{X}$.

Indeed, for any normal singular cubic surface $X$ with no parameters, we can show that $\Psi$ is surjective, so we have an exact sequence

$$
0 \longrightarrow \operatorname{Ker} \Psi \longrightarrow \text { Aut } \tilde{X} \longrightarrow \text { Aut } \Gamma_{\tilde{X}} \longrightarrow 0 \text {. }
$$

Ker $\Psi$ is determined by the geometry on a projective plane $\mathbf{P}^{2}$, and Aut $\Gamma_{\tilde{X}}$ is a finite group determined by the intersection relations of $\Gamma_{\tilde{X}}$. Hereafter, we determine Aut $\tilde{X}$ with respect to each type of singularity by investigating the properties of $\Psi$ and the group structures of $\operatorname{Ker} \Psi$ and $\operatorname{Aut} \Gamma_{\tilde{X}}$.

First we recall a well-known fact below. It is useful to determine automorphism groups on normal singular cubic surfaces which have $A_{1}$ singularity. 
Lemma 4.3. Let $C$ be a smooth conic on $\mathbf{P}^{2}$. Then, the subgroup $G:=\{\tau \in$ Aut $\mathbf{P}^{2} \mid \tau(C)=C$ \} of Aut $\mathbf{P}^{2}$ is isomorphic to Aut $\mathbf{P}^{1}$.

Proof. See Harris [7, pp. 117-118.

4.2. Type $A_{1} A_{3}$. We use the notation and conditions in subsection 2.2.5. First we determine Aut $\Gamma_{\tilde{X}}$. For any $s \in \operatorname{Aut} \Gamma_{\tilde{X}}$, we have $s\left(\tilde{C}_{2}\right)=\tilde{C}_{2}, s\left(\tilde{E}_{i}\right)=\tilde{E}_{i}(i=$ $1,2,3,4), s\left(\tilde{L}_{1,2}\right)=\tilde{L}_{1,2}, s\left(\tilde{L}_{5,6}\right)=\tilde{L}_{5,6}$. On the other hand, $s\left(\tilde{E}_{5}\right)$ is either $\tilde{E}_{5}$ or $\tilde{E}_{6}$. If $s\left(\tilde{E}_{5}\right)=\tilde{E}_{5}$, then $s\left(\tilde{L}_{1,5}\right)=\tilde{L}_{1,5}, s\left(\tilde{L}_{1,6}\right)=\tilde{L}_{1,6}$ and $s\left(\tilde{E}_{6}\right)=\tilde{E}_{6}$. Hence, $s=\operatorname{id}_{\Gamma_{\tilde{X}}}$. On the other hand, if $s\left(\tilde{E}_{5}\right)=\tilde{E}_{6}$, then $s\left(\tilde{L}_{1,5}\right)=\tilde{L}_{1,6}, s\left(\tilde{L}_{1,6}\right)=\tilde{L}_{1,5}$ and $s\left(\tilde{E}_{6}\right)=\tilde{E}_{5}$. Hence, $s \neq \operatorname{id}_{\Gamma_{\tilde{X}}}$ and $s^{2}=\operatorname{id}_{\Gamma_{\tilde{X}}}$. Therefore, we have Aut $\Gamma_{\tilde{X}} \simeq \mathbf{Z} / 2 \mathbf{Z}$.

Let $s_{1}$ be the element of order 2 in Aut $\Gamma_{\tilde{X}}$. We secondly prove that $\Psi$ is surjective, by finding $\tilde{\sigma} \in$ Aut $\tilde{X}$ with $\Psi(\tilde{\sigma})=s_{1}$. There exists a unique automorphism $\sigma$ on $\mathbf{P}^{2}$ with $\sigma\left(C_{2}\right)=C_{2}, \sigma\left(P_{1}\right)=P_{1}, \sigma\left(P_{5}\right)=P_{6}, \sigma\left(P_{6}\right)=P_{5}$ by Lemma 4.3, $\sigma$ induces $\tilde{\sigma} \in$ Aut $\tilde{X}$ such that $\Psi(\tilde{\sigma})=s_{1}$ by Lemma 3.1] hence $\Psi$ is surjective.

We finally prove that $\Psi$ is injective, i.e., $\operatorname{Ker} \Psi=\{$ id $\}$. For $\tilde{\sigma} \in \operatorname{Ker} \Psi, \tilde{\sigma}$ induces $\sigma \in \operatorname{Aut} \mathbf{P}^{2}$ via $\varepsilon: \tilde{X} \rightarrow \mathbf{P}^{2}$ by Lemma 3.3 . Then, $\sigma\left(C_{2}\right)=C_{2}, \sigma\left(P_{1}\right)=P_{1}, \sigma\left(P_{5}\right)=$ $P_{5}$ and $\sigma\left(P_{6}\right)=P_{6}$, so $\sigma=\operatorname{id}_{\mathbf{P}^{2}}$ by Lemma 4.3 and we have $\tilde{\sigma}=\operatorname{id}_{\tilde{X}}$. Therefore, $\Psi$ is injective. Hence, Aut $\tilde{X} \simeq \mathbf{Z} / 2 \mathbf{Z}$.

For $X=\left\{\left(x_{0} x_{2}-x_{1}^{2}\right) x_{3}-\left(x_{0}-x_{1}\right)\left(-x_{1}+x_{2}\right)\left(x_{0}-2 x_{1}+x_{2}\right)=0\right\}$ in subsection 3.2.5, Aut $X$ is generated by

$$
X \ni\left(x_{0}: x_{1}: x_{2}: x_{3}\right) \mapsto\left(x_{2}: x_{1}: x_{0}: x_{3}\right) \in X .
$$

4.3. Type $2 A_{1} A_{2}$. We use the notation and conditions in subsection 2.2.6. In the same way as above, Aut $\Gamma_{\tilde{X}} \simeq \mathbf{Z} / 2 \mathbf{Z}$, and $\Psi$ is injective. We prove that $\Psi$ is surjective. Let $s_{1}$ be the element of order 2 in Aut $\Gamma_{\tilde{X}}$. Then, we have $s_{1}\left(\tilde{C}_{2}\right)=$ $\tilde{E}_{4}, s_{1}\left(\tilde{E}_{4}\right)=\tilde{C}_{2}, s_{1}\left(\tilde{E}_{5}\right)=\tilde{E}_{5}, s_{1}\left(\tilde{L}_{4,5}\right)=\tilde{L}_{4,5}, s_{1}\left(\tilde{L}_{1,4}\right)=\tilde{E}_{3}, s_{1}\left(\tilde{E}_{3}\right)=\tilde{L}_{1,4}$, $s_{1}\left(\tilde{E}_{1}\right)=\tilde{E}_{2}, s_{1}\left(\tilde{E}_{2}\right)=\tilde{E}_{1}, s_{1}\left(\tilde{L}_{1,6}\right)=\tilde{L}_{1,2}, s_{1}\left(\tilde{L}_{1,2}\right)=\tilde{L}_{1,6}, s_{1}\left(\tilde{E}_{6}\right)=\tilde{L}_{4,6}$ and $s_{1}\left(\tilde{L}_{4,6}\right)=\tilde{E}_{6}$. We have a birational morphism $p: \tilde{X} \rightarrow \mathbf{P}^{2}$ which is a succession of blowings-down of the curves $\tilde{L}_{4,6}, \tilde{E}_{5}, \tilde{C}_{2}, \tilde{L}_{1,4}, \tilde{E}_{1}$ and $\tilde{E}_{2}$, in that order. Let $C^{\prime}:=$ $p\left(\tilde{E}_{4}\right), Q_{1}:=p\left(\tilde{L}_{1,4}\right)=p\left(\tilde{E}_{1}\right)=p\left(\tilde{E}_{2}\right), Q_{4}:=p\left(\tilde{C}_{2}\right)=p\left(\tilde{E}_{5}\right)$ and $Q_{6}:=p\left(\tilde{L}_{4,6}\right)$. Then, $C^{\prime}$ is a smooth conic which contains three points $Q_{1}, Q_{4}, Q_{6}$. By Lemma 3.4. there exists a unique automorphism $\sigma$ on $\mathbf{P}^{2}$ with $\sigma\left(C_{2}\right)=C^{\prime}, \sigma\left(P_{1}\right)=Q_{1}$, $\sigma\left(P_{4}\right)=Q_{4}$ and $\sigma\left(P_{6}\right)=Q_{6} . \sigma$ induces $\tilde{\sigma} \in$ Aut $\tilde{X}$ by Lemma 3.1. and we have $\Psi(\tilde{\sigma})=s_{1}$. Hence, Aut $\tilde{X} \simeq \mathbf{Z} / 2 \mathbf{Z}$. by

For $X=\left\{\left(x_{0} x_{2}-x_{1}^{2}\right) x_{3}-x_{1}^{2}\left(x_{0}-x_{1}\right)=0\right\}$ in subsection 3.2.6. Aut $X$ is generated

$$
X \ni\left(x_{0}: x_{1}: x_{2}: x_{3}\right) \mapsto\left(x_{3}: x_{1}: x_{2}: x_{0}\right) \in X .
$$

4.4. Type $4 A_{1}$. We use the notation and conditions in subsection 2.2.7 In the same way as above, Aut $\Gamma_{\tilde{X}} \simeq \Sigma_{4}$, and $\Psi$ is injective. Let $F_{1}:=\tilde{C}_{2}, F_{2}:=\tilde{E}_{1}$, $F_{3}:=\tilde{E}_{3}$ and $F_{4}:=\tilde{E}_{5} . \quad s \in$ Aut $\Gamma_{\tilde{X}}$ induces $\bar{s} \in \Sigma_{4}$ satisfying $s\left(F_{i}\right)=F_{\bar{s}(i)}$ for $i=1,2,3,4$. For each $i=2,3,4$, there exists a unique $(-1)$-curve $G_{i}$ meeting both $F_{\bar{s}(1)}$ and $F_{\bar{s}(i)}$. We have a birational morphism $p: \tilde{X} \rightarrow \mathbf{P}^{2}$ which is a succession of blowings-down of $G_{4}, F_{\bar{s}(4)}, G_{3}, F_{\bar{s}(3)}, G_{2}$ and $F_{\bar{s}(2)}$, in that order. Let $C^{\prime}:=p\left(F_{\bar{s}(1)}\right)$ and $Q_{i}:=p\left(G_{i}\right)=p\left(F_{\bar{s}(i)}\right)(i=2,3,4)$. Then, $C^{\prime}$ is a smooth conic which contains three points, $Q_{2}, Q_{3}, Q_{4}$. There exists a unique automorphism 
$\sigma$ on $\mathbf{P}^{2}$ with $\sigma\left(C_{2}\right)=C^{\prime}, \sigma\left(P_{1}\right)=Q_{2}, \sigma\left(P_{3}\right)=Q_{3}$ and $\sigma\left(P_{5}\right)=Q_{4} . \sigma$ induces $\tilde{\sigma} \in$ Aut $\tilde{X}$ by Lemma 3.1, and $\tilde{\sigma}$ satisfies $\Psi(\tilde{\sigma})=s$. Hence, Aut $\tilde{X} \simeq \Sigma_{4}$.

For $X=\left\{\left(x_{0} x_{2}-x_{1}^{2}\right) x_{3}-\left(x_{0}-x_{1}\right)\left(x_{1}-x_{2}\right) x_{1}=0\right\}$ in subsection 3.2.7. Aut $X$ is generated by

$$
\begin{gathered}
X \ni\left(x_{0}: x_{1}: x_{2}: x_{3}\right) \mapsto\left(x_{0}: x_{0}-x_{1}: x_{0}-2 x_{1}+x_{2}: x_{3}\right) \in X, \\
X \ni\left(x_{0}: x_{1}: x_{2}: x_{3}\right) \mapsto\left(x_{0}-2 x_{1}+x_{2}:-x_{1}+x_{2}: x_{2}: x_{3}\right) \in X,
\end{gathered}
$$

and

$$
X \ni\left(x_{0}: x_{1}: x_{2}: x_{3}\right) \mapsto\left(x_{0}: x_{1}: x_{1}+x_{3}:-x_{1}+x_{2}\right) \in X .
$$

4.5. Type $A_{1} A_{4}$. We use the notation and conditions in subsection 2.2.8, In the same way as above, we have Aut $\Gamma_{\tilde{X}}=\{\operatorname{id}\}$. So Aut $\tilde{X}$ is isomorphic to $\operatorname{Ker} \Psi$. $\tilde{\sigma} \in \operatorname{Ker} \Psi$ induces an automorphism $\sigma$ on $\mathbf{P}^{2}$ with $\sigma\left(P_{1}\right)=P_{1}$ and $\sigma\left(P_{6}\right)=P_{6}$, and we have a group homomorphism $\psi: \operatorname{Ker} \Psi \rightarrow$ Aut $\mathbf{P}^{2}$ which is injective. It is easy to see that $\operatorname{Im} \psi=\left\{\sigma \in \operatorname{Aut} \mathbf{P}^{2} \mid \sigma\left(C_{2}\right)=C_{2}, \sigma\left(P_{1}\right)=P_{1}, \sigma\left(P_{6}\right)=P_{6}\right\}$. This subgroup of Aut $\mathbf{P}^{2}$ is isomorphic to a subgroup of Aut $\mathbf{P}^{1}$ which consists of all automorphisms leaving two points fixed, and it is isomorphic to $\mathbf{C}^{\times}$. Hence, Aut $\tilde{X} \simeq \mathbf{C}^{\times}$.

For $X=\left\{\left(x_{0} x_{2}-x_{1}^{2}\right) x_{3}-x_{0}^{2} x_{1}=0\right\}$ in subsection 3.2.8, any element of Aut $X$ is written by

$$
X \ni\left(x_{0}: x_{1}: x_{2}: x_{3}\right) \mapsto\left(a x_{0}: a^{2} x_{1}: a^{3} x_{2}: x_{3}\right) \in X,
$$

where $a \in \mathbf{C}^{\times}$.

4.6. Type $2 A_{1} A_{3}$. We use the notation and conditions in subsection 2.2.9, Similarly, we have Aut $\Gamma_{\tilde{X}} \simeq \mathbf{Z} / 2 \mathbf{Z}$. Let $s_{1}$ be an element of order 2 in Aut $\Gamma_{\tilde{X}}$. In the same way as above, we get $\tilde{\sigma} \in$ Aut $\tilde{X}$ with $\Psi(\tilde{\sigma})=s_{1}$, so $\Psi$ is surjective. Therefore, we have the short exact sequence of groups $(\sharp)$. For $X=\left\{\left(x_{0} x_{2}-x_{1}^{2}\right) x_{3}-x_{0} x_{1}^{2}=0\right\}$ in subsection 3.2.9, $\tau \in$ Aut $X$ induced by $\tilde{\sigma}$ is written by

$$
\tau: X \ni\left(x_{0}: x_{1}: x_{2}: x_{3}\right) \mapsto\left(x_{3}: x_{1}: x_{2}: x_{0}\right) \in X .
$$

We define a group homomorphism $\theta:$ Aut $\Gamma_{\tilde{X}} \rightarrow$ Aut $\tilde{X}$ by $\theta($ id $)=$ id and $\theta\left(s_{1}\right)=\tilde{\sigma}$. Then, the exact sequence $(\sharp)$ splits by a section $\theta$. In the same way as section 4.5. we have $\operatorname{Ker} \Psi \simeq \mathbf{C}^{\times}$. Hence, Aut $\tilde{X} \simeq \mathbf{C}^{\times} \rtimes \mathbf{Z} / 2 \mathbf{Z}$, and Aut $X$ is generated by $\tau$ and

$$
X \ni\left(x_{0}: x_{1}: x_{2}: x_{3}\right) \mapsto\left(x_{0}: a x_{1}: a^{2} x_{2}: x_{3}\right) \in X,
$$

where $a \in \mathbf{C}^{\times}$.

4.7. Type $A_{1} 2 A_{2}$. We use the notation and conditions in subsection 2.2.10, In the same way as section 4.6, we have Aut $\tilde{X} \simeq \mathbf{C}^{\times} \rtimes \mathbf{Z} / 2 \mathbf{Z}$. For $X=\left\{\left(x_{0} x_{2}-x_{1}^{2}\right) x_{3}-\right.$ $\left.x_{1}^{3}=0\right\}$ in subsection 3.2.10, Aut $X$ is generated by

$$
X \ni\left(x_{0}: x_{1}: x_{2}: x_{3}\right) \mapsto\left(x_{2}: x_{1}: x_{0}: x_{3}\right) \in X
$$

and

$$
X \ni\left(x_{0}: x_{1}: x_{2}: x_{3}\right) \mapsto\left(a^{2} x_{0}: a x_{1}: x_{2}: a x_{3}\right) \in X,
$$

where $a \in \mathbf{C}^{\times}$. 
4.8. Type $A_{1} A_{5}$. We use the notation and conditions in subsection 2.2.11. Clearly, Aut $\Gamma_{\tilde{X}}=\{$ id $\}$, so we have

$$
\text { Aut } \tilde{X} \simeq \operatorname{Ker} \Psi \simeq\left\{\sigma \in \operatorname{Aut} \mathbf{P}^{1} \mid \sigma(P)=P\right\},
$$

where $P \in \mathbf{P}^{1}$ is a point. The right hand is isomorphic to

$$
H:=\left\{\left(\begin{array}{ll}
1 & 0 \\
s & t
\end{array}\right) \in G L_{2}(\mathbf{C})\right\},
$$

where $G L_{2}(\mathbf{C})$ is the general linear group of dimension 2. We have a natural split exact sequence for $H$, so $H \simeq \mathbf{C} \rtimes \mathbf{C}^{\times}$. Hence, Aut $\tilde{X} \simeq \mathbf{C} \rtimes \mathbf{C}^{\times}$. For $X=\left\{\left(x_{0} x_{2}-x_{1}^{2}\right) x_{3}-x_{0}^{3}=0\right\}$ in subsection 3.2.11, Aut $X$ is generated by

$$
\begin{aligned}
X \ni\left(x_{0}: x_{1}: x_{2}: x_{3}\right) & \\
& \mapsto\left(a^{2} x_{0}: a^{2} b x_{0}+a^{3} x_{1}: a^{2} b^{2} x_{0}+2 a^{3} b x_{1}+a^{4} x_{2}: x_{3}\right) \in X,
\end{aligned}
$$

where $a, b \in \mathbf{C}$ and $a \neq 0$.

4.9. Type $3 A_{2}$. We use the notation and conditions in subsection 2.3.6. Clearly, Aut $\Gamma_{\tilde{X}} \simeq \Sigma_{3}$ and

$$
\operatorname{Ker} \Psi \simeq\left\{\sigma \in \operatorname{Aut} \mathbf{P}^{2} \mid \sigma\left(Q_{i}\right)=Q_{i}(i=1,2,3)\right\},
$$

where $Q_{1}, Q_{2}, Q_{3}$ are points and are not collinear. The right hand is isomorphic to $\left(\mathbf{C}^{\times}\right)^{2}$. For $X=\left\{x_{0} x_{1} x_{3}-x_{2}^{3}=0\right\}$ in subsection 3.3.6.

$$
\begin{aligned}
& \tau_{1}: X \ni\left(x_{0}: x_{1}: x_{2}: x_{3}\right) \mapsto\left(x_{1}: x_{0}: x_{2}: x_{3}\right) \in X, \\
& \tau_{2}: X \ni\left(x_{0}: x_{1}: x_{2}: x_{3}\right) \mapsto\left(x_{0}: x_{3}: x_{2}: x_{1}\right) \in X
\end{aligned}
$$

are elements of Aut $X$. Let $\tilde{\sigma}_{i}$ be the elements of Aut $\tilde{X}$ corresponding to $\tau_{i}$, and $s_{i}:=\Psi\left(\tilde{\sigma}_{i}\right)$. Then $s_{1}, s_{2}$ generate Aut $\Gamma_{\tilde{X}}$, so $\Psi$ is surjective. On the other hand, we can define a natural group homomorphism $\theta:$ Aut $\Gamma_{\tilde{X}} \rightarrow$ Aut $\tilde{X}$, which is injective. So the exact sequence $(\sharp)$ splits by a section $\theta$. Hence, Aut $X \simeq\left(\mathbf{C}^{\times}\right)^{2} \rtimes \Sigma_{3}$. Aut $X$ is generated by $\tau_{1}, \tau_{2}$ and

$$
X \ni\left(x_{0}: x_{1}: x_{2}: x_{3}\right) \mapsto\left(a^{2} b x_{0}: a b^{2} x_{1}: a b x_{2}: x_{3}\right) \in X,
$$

where $a, b \in \mathbf{C}^{\times}$.

4.10. Type $A_{4}$. We use the notation and conditions in subsection 2.3.10. Clearly, Aut $\Gamma_{\tilde{X}} \simeq \mathbf{Z} / 2 \mathbf{Z}$, and $\Psi$ is surjective. So we have the short exact sequence $(\sharp)$. We can put $C_{2}=\left\{f_{2}=x_{0} x_{1}=0\right\}, L=\left\{x_{0}=0\right\}, L^{\prime}=\left\{x_{1}=0\right\}, C_{3}=\left\{f_{3}=\right.$ $\left.x_{0}^{2} x_{2}+x_{1}^{3}-x_{1} x_{2}^{2}=0\right\}$ and $X=\left\{x_{3} f_{2}-f_{3}=0\right\}$ by subsection 3.3.10, For

$$
\iota: X \ni\left(x_{0}: x_{1}: x_{2}: x_{3}\right) \mapsto\left(x_{0}: i x_{1}:-i x_{2}:-x_{3}\right) \in X \text {, }
$$

where $i=\sqrt{-1}, \Psi(\iota)$ is an element of order 2 of Aut $\Gamma_{\tilde{X}}$. On the other hand, $\operatorname{Ker} \Psi$ is isomorphic to a subgroup of Aut $\mathbf{P}^{2}$ which fixes $L=L_{1,2,4}, L^{\prime}=L_{3,4,5}$, $P_{1}=(0: 1: 1), P_{2}=(0: 1:-1), P_{3}=(1: 0: 0)$ and $P_{6}$. We lift up automorphisms on $\mathbf{P}^{2}$ to $\tilde{X}$, and apply conditions above. We have $\operatorname{Ker} \Psi \simeq \mathbf{Z} / 2 \mathbf{Z}$, and it is generated by

$$
X \ni\left(x_{0}: x_{1}: x_{2}: x_{3}\right) \mapsto\left(x_{0}:-x_{1}:-x_{2}: x_{3}\right) \in X,
$$

via $\gamma:$ Aut $X \simeq$ Aut $\tilde{X}$. Therefore, Aut $X$ is a finite group of order 4 . Since $\iota$ is an element of order 4 in Aut $X$, we have Aut $X \simeq \mathbf{Z} / 4 \mathbf{Z}$. 
4.11. Type $A_{5}$. We use the notation and conditions in subsection 2.3.12 It is easy to see that Aut $\Gamma_{\tilde{X}} \simeq \mathbf{Z} / 2 \mathbf{Z}$. We can put $C_{2}=\left\{f_{2}=x_{0} x_{1}=0\right\}, L=\left\{x_{0}=\right.$ $0\}, L^{\prime}=\left\{x_{1}=0\right\}, C_{3}=\left\{f_{3}=x_{0}^{3}+x_{1}^{3}-x_{1} x_{2}^{2}=0\right\}$ and $X=\left\{x_{3} f_{2}-f_{3}=0\right\}$ by subsection 3.3.12, For

$$
\tau: X \ni\left(x_{0}: x_{1}: x_{2}: x_{3}\right) \mapsto\left(x_{0}: x_{1}:-x_{2}: x_{3}\right) \in X
$$

$\Psi(\tau)$ is an element of order 2 in $\operatorname{Aut} \Gamma_{\tilde{X}}$, so we have the short exact sequence $(\sharp)$ which splits. On the other hand, Ker $\Psi$ is isomorphic to a subgroup of Aut $\mathbf{P}^{2}$ which fixes $L=L_{1,2,3}, L^{\prime}=L_{3,4,5}, P_{1}=(0: 1: 1), P_{2}=(0: 1:-1)$ and $P_{6}$. In the same way as above, $\operatorname{Ker} \Psi$ is generated by

$$
\begin{aligned}
\sigma: X \ni\left(x_{0}: x_{1}: x_{2}\right. & \left.: x_{3}\right) \\
& \mapsto\left(x_{0}: \omega^{i} x_{1}: a x_{0}+\omega^{i} x_{2}:-a^{2} x_{0}-2 \omega^{i} a x_{2}+\omega^{-i} x_{3}\right) \in X,
\end{aligned}
$$

via $\gamma:$ Aut $X \simeq$ Aut $\tilde{X}$, where $a \in \mathbf{C}, \omega^{3}=1(\omega \neq 1), i=0,1,2$. It is isomorphic to $\mathbf{C} \rtimes \mathbf{Z} / 3 \mathbf{Z}$. Hence, Aut $X \simeq(\mathbf{C} \rtimes \mathbf{Z} / 3 \mathbf{Z}) \rtimes \mathbf{Z} / 2 \mathbf{Z}$.

4.12. Type $D_{4}$. We use the notation and conditions in subsection 2.4.1. It is easy to see that Aut $\Gamma_{\tilde{X}} \simeq \Sigma_{3}$. There are two normal forms in this case (cf. subsection 3.4.1).

(1) In the case of $C_{2}=\left\{f_{2}=x_{0}^{2}=0\right\}$ and $C_{3}=\left\{f_{3}=x_{1}^{3}+x_{2}^{3}=0\right\}, \operatorname{Ker} \Psi$ is isomorphic to a subgroup of Aut $\mathbf{P}^{2}$ which fixes $P_{1}, P_{3}, P_{5}, L_{1,2}, L_{3,4}$ and $L_{5,6}$, where $L_{1,2}, L_{3,4}$ and $L_{5,6}$ meet at a point. Hence, $\operatorname{Ker} \Psi \simeq \mathbf{C}^{\times}$. Similarly, we have the exact sequence $(\sharp)$ which splits, so we have Aut $X \simeq$ $\mathbf{C}^{\times} \rtimes \Sigma_{3}$, and it is generated by

$$
\begin{gathered}
X \ni\left(x_{0}: x_{1}: x_{2}: x_{3}\right) \mapsto\left(x_{0}: x_{2}: x_{1}: x_{3}\right) \in X, \\
X \ni\left(x_{0}: x_{1}: x_{2}: x_{3}\right) \mapsto\left(x_{0}: \omega x_{2}: \omega^{2} x_{1}: x_{3}\right) \in X
\end{gathered}
$$

and

$$
X \ni\left(x_{0}: x_{1}: x_{2}: x_{3}\right) \mapsto\left(a^{3} x_{0}: a^{2} x_{1}: a^{2} x_{2}: x_{3}\right) \in X,
$$

where $a \in \mathbf{C}^{\times}$.

(2) In the case of $C_{2}=\left\{f_{2}=x_{0}^{2}=0\right\}$ and $C_{3}=\left\{f_{3}=x_{1}^{3}+x_{2}^{3}+x_{0} x_{1} x_{2}=0\right\}$, we have $\operatorname{Ker} \Psi=\{\mathrm{id}\}$. Since automorphisms

$$
X \ni\left(x_{0}: x_{1}: x_{2}: x_{3}\right) \mapsto\left(x_{0}: x_{2}: x_{1}: x_{3}\right) \in X
$$

and

$$
X \ni\left(x_{0}: x_{1}: x_{2}: x_{3}\right) \mapsto\left(x_{0}: \omega x_{2}: \omega^{2} x_{1}: x_{3}\right) \in X
$$

correspond to generators of Aut $\Gamma_{\tilde{X}} \simeq \Sigma_{3}, \Psi$ is surjective. Hence, Aut $X \simeq$ $\Sigma_{3}$.

4.13. Type $D_{5}$. We use the notation and conditions in subsection 2.4.2. We can put $C_{2}=\left\{x_{0}^{2}=0\right\}, L=\left\{x_{0}=0\right\}, C_{3}=\left\{x_{0} x_{2}^{2}+x_{1}^{2} x_{2}=0\right\}$ and $X=\left\{x_{3} f_{2}-f_{3}=0\right\}$ by subsection 3.4.2. Clearly, Aut $\Gamma_{\tilde{X}}=\{\mathrm{id}\}$, and $\operatorname{Ker} \Psi$ is isomorphic to a subgroup of Aut $\mathbf{P}^{2}$ which fixes $L=L_{1,2,5}, L_{5,6}, P_{1}=(0: 0: 1), P_{3}$ and $P_{4}$. In the same way as above, we have $\operatorname{Ker} \Psi \simeq \mathbf{C}^{\times}$. Hence, Aut $X \simeq \mathbf{C}^{\times}$, and it is generated by

$$
X \ni\left(x_{0}: x_{1}: x_{2}: x_{3}\right) \mapsto\left(a^{4} x_{0}: a^{3} x_{1}: a^{2} x_{2}: x_{3}\right) \in X,
$$

where $a \in \mathbf{C}^{\times}$. 
4.14. Type $E_{6}$. We use the notation and conditions in subsection 2.4.3. We can put $C_{2}=\left\{x_{0}^{2}=0\right\}, L=\left\{x_{0}=0\right\}, C_{3}=\left\{x_{0} x_{2}^{2}+x_{1}^{3}=0\right\}$ and $X=\left\{x_{3} f_{2}-f_{3}=0\right\}$ by subsection 3.4.3. Clearly, Aut $\Gamma_{\tilde{X}}=\{\mathrm{id}\}$, and $\operatorname{Ker} \Psi$ is isomorphic to a subgroup of Aut $\mathbf{P}^{2}$ which fixes $L=L_{1,2,3}, P_{1}=(0: 0: 1), P_{4}, P_{5}$ and $P_{6}$. In the same way as above, we have $\operatorname{Ker} \Psi \simeq \mathbf{C} \rtimes \mathbf{C}^{\times}$. Hence, Aut $X \simeq \mathbf{C} \rtimes \mathbf{C}^{\times}$, and it is generated by

$$
X \ni\left(x_{0}: x_{1}: x_{2}: x_{3}\right) \mapsto\left(a^{6} x_{0}: a^{4} x_{1}: a^{3} b x_{0}+a^{3} x_{2}: b^{2} x_{0}+2 b x_{2}+x_{3}\right) \in X,
$$

where $a \in \mathbf{C}^{\times}, b \in \mathbf{C}$.

\section{REFERENCES}

1. A. Beauville, Complex Algebraic Surfaces. London Mathematical Society Lecture Note Series 68. Cambridge Univ. Press, 1983. MR732439 (85a:14024)

2. J. W. Bruce and C. T. C. Wall, On the classification of cubic surfaces. J. London Math. Soc. (2) 19 (1979), no. 2, 245-256. MR533323 (80f:14021)

3. A. Cayley, A memoir on cubic surfaces. Phil. Trans. Roy. Soc., 159 (1869), 231-326.

4. D. F. Coray and M. A. Tsfasman, Arithmetic on singular Del Pezzo surfaces. Proc. London Math. Soc. (3) 57 (1988), no. 1, 25-87. MR940430 (89f:11083)

5. M. Demazure, Surfaces de del Pezzo II-V. Séminaire sur les Singularités des Surfaces, Lecture Notes in Math., Vol. 777, Springer-Verlag, Berlin-Heidelberg-New York, 1980. MR579026 (82d:14021)

6. I. V. Dolgachev and V. A. Iskovskikh, Finite subgroups of the plane Cremona group. arXiv:math/0610595v2 [math.AG] 21 Jul 2007.

7. J. Harris, Algebraic geometry. Graduate Texts in Mathematics, 133, Springer-Verlag, New York, 1995. MR 1416564(97e:14001)

8. R. Hartshorne, Algebraic Geometry. Graduate Texts in Mathematics, No. 52. Springer-Verlag, New York-Heidelberg, 1977. MR0463157 (57:3116)

9. T. Hosoh, Automorphism groups of cubic surfaces. J. Algebra 192 (1997), no. 2, 651-677. MR 1452681 (99d:14042)

10. T. Hosoh, Automorphism groups of quartic del Pezzo surfaces. J. Algebra 185 (1996), no. 2, 374-389. MR1417377 (97i:14026)

11. S. Iitaka, Algebraic Geometry. Graduate Texts in Mathematics, 76. Springer-Verlag, New York-Berlin, 1982. MR637060 (84j:14001)

12. Y. Kawamata, Algebraic Varieties. Kyoritsu Shuppan Co., Ltd., 1997.

13. M. Koitabashi, Automorphism groups of generic rational surfaces. J. Algebra 116 (1988), no. 1, 130-142. MR944150 (89f:14045)

14. M. Reid, Young person's guide to canonical singularities. Proc. Sympos. Pure Math, 461(1987), 345-414. MR927963 (89b:14016)

15. Y. Manin, Cubic Forms: Algebra, Geometry, Arithmetic. North-Holland, Amsterdam, 1974. MR833513(87d:11037)

16. L. Schläfli, On the distribution of surfaces of the third order into species. Phil. Trans. Roy. Soc., 153 (1864), 193-247.

17. B. Segre, The Non-singular Cubic Surfaces. Oxford University Press, Oxford, 1942. $\operatorname{MR} 0008171(4: 254 \mathrm{~b})$

Graduate School of Mathematics, Kyushu University, Hakozaki 6-10-1, Higashi-ku, FUKUOKA, 812-8581, JAPAN

E-mail address: sakamaki@math.kyushu-u.ac.jp

Current address: System Engineering Laboratory, Toshiba Corporate Research \& Development

Center, 1, Komukai Toshiba-cho, Saiwai-ku, Kawasaki-shi, Kanagawa, 212-8582, Japan

E-mail address: yoshiyuki.sakamaki@toshiba.co.jp 